\title{
A new viscosity-type iteration for a finite family of split variational inclusion and fixed point problems between Hilbert and Banach spaces
}

\author{
C. Izuchukwu' ${ }^{1}$ F.O. Isiogugu $u^{1,2,3^{*}}$ and C.C. Okeke $e^{1,2}$
}

\author{
"Correspondence: \\ felicia.isiogugu@unn.edu.ng \\ 'School of Mathematics, Statistics \\ and Computer Science, University of \\ Kwazulu-Natal, Durban, South Africa \\ ${ }^{2}$ DST-NRF Center of Excellence in \\ Mathematical and Statistical \\ Sciences (CoE-Mass), Johannesburg, \\ South Africa \\ Full list of author information is \\ available at the end of the article
}

\begin{abstract}
In this paper, we introduce a new viscosity-type iteration process for approximating a common solution of a finite family of split variational inclusion problem and fixed point problem. We prove that the proposed algorithm converges strongly to a common solution of a finite family of split variational inclusion problems and fixed point problem for a finite family of type-one demicontractive mappings between a Hilbert space and a Banach space. Furthermore, we applied our results to study a finite family of split convex minimization problems, and also considered a numerical experiment of our results to further illustrate its applicability. Our results extend and improve the results of Byrne et al. (J. Nonlinear Convex Anal. 13:759-775, 2012), Kazmi and Rizvi (Optim. Lett. 8(3):1113-1124, 2014), Moudafi (J. Optim. Theory Appl. 150:275-283, 2011), Shehu and Ogbuisi (Rev. R. Acad. Cienc. Exactas Fís. Nat., Ser. A Mat. 110(2):503-518, 2016), Takahashi and Yao (Fixed Point Theory Appl. 2015:87, 2015), Chidume and Ezeora (Fixed Point Theory Appl. 2014:111, 2014), and a host of other important results in this direction.
\end{abstract}

MSC: 47H09; 47H10;49J20; 49J40

Keywords: Variational inclusion problem; Viscosity iterative method; Convex minimization problem; Strictly pseudocontractions; Maximal monotone mapping; Resolvent operators

\section{Introduction}

Let $H$ be a real Hilbert space, $M: H \rightarrow 2^{H}$ be any multivalued mapping and $N: H \rightarrow H$ be a single-valued mapping. The variational inclusion problem (VIP) is the problem of finding $x^{*} \in H$ such that

$$
0 \in M\left(x^{*}\right)+N\left(x^{*}\right)
$$

One of the most popular and effective methods for solving the VIP (1.1) is the following forward-backward splitting method which includes the proximal point method and the gradient method (see $[11,26])$ : For any fixed $x_{1} \in H$ and $\lambda>0$ :

$$
x_{n+1}=(I+\lambda M)^{-1}(I-\lambda N) x_{n}, \quad n \geq 1 .
$$

(c) The Author(s) 2019. This article is distributed under the terms of the Creative Commons Attribution 4.0 International License (http://creativecommons.org/licenses/by/4.0/), which permits unrestricted use, distribution, and reproduction in any medium, provided you give appropriate credit to the original author(s) and the source, provide a link to the Creative Commons license, and indicate if changes were made. 
If $N \equiv 0$ in (1.1), then the VIP reduces to the following null point problem (NPP):

Find $x^{*} \in H$ such that $0 \in M x^{*}$.

When $M$ is monotone, problems (1.1) and (1.2) are known to be the most important problems in monotone operator theory, nonlinear and convex analysis, due to the role they play in optimization, variational inequalities, semi group theory and evolution equations, among others. Moreover, many mathematical problems such as optimization problems, equilibrium problems, variational inequality problems, saddle point problems, etc., can be modeled as (1.2) or more generally, (1.1) (see [8, 15, 23, 29, 37]). Thus, VIPs and NPPs are of central importance in nonlinear and convex analysis. As a result of this, many authors have studied VIPs and NPPs in both Hilbert and Banach spaces (see [1, 2, 10, 11, 26, 28] and the references therein).

The study of VIPs and NPPs has been extended to the study of split variational inclusion problem (SVIP) and split common null point problem (SCNPP), respectively. The development of these split-type problems can be traced to the split feasibility problem (SFP), introduced by Censor and Elfving [9] as follows: Find

$$
x^{*} \in C \text { such that } y^{*}=A x^{*} \in Q \text {, }
$$

where $C$ and $Q$ are nonempty closed and convex subsets of $\mathbb{R}^{n}$ and $\mathbb{R}^{m}$, respectively, and $A$ is an $m \times n$ real matrix. The SFP is well known to have wide applications in many fields such as phase retrieval, medical image reconstruction, signal processing, radiation therapy treatment planning, among others (see [7, 9] and the references therein). The SFP was also studied by Byrne [6] using the following CQ-iterative algorithm:

$$
x_{n+1}=P_{C}\left(I-\gamma A^{*}\left(I-P_{Q}\right) A\right) x_{n}, \quad n \in \mathbb{N},
$$

where $\gamma \in\left(0, \frac{2}{\lambda}\right)$ with $\lambda$ being the spectral radius of the operator $A^{*} A$. Byrne [6] proved that the sequence generated by Algorithm (1.4) converges weakly to a solution of (1.3).

In 2012, Byrne et al. [8] extended the NPP (1.2) to the following SCNPP: Find $x^{*} \in H_{1}$ such that

$$
0 \in M_{1}\left(x^{*}\right)
$$

and

$$
y^{*}=A x^{*} \in H_{2} \text { such that } 0 \in M_{2}\left(y^{*}\right),
$$

where $H_{1}$ and $H_{2}$ are two real Hilbert spaces, $M_{1}$ and $M_{2}$ are two multivalued maximal monotone mappings and $A$ is a bounded linear operator from $H_{1}$ to $H_{2}$. Byrne et al. [8] proposed the following algorithm to solve problem (1.5)-(1.6): For a given $x_{1} \in H_{1}$, the sequence $\left\{x_{n}\right\}$ is given by

$$
x_{n+1}=J_{\lambda}^{M_{1}}\left(x_{n}+\gamma A^{*}\left(J_{\lambda}^{M_{2}}-I\right) A x_{n}\right), \quad \lambda>0,
$$


where $J_{\lambda}^{M_{1}}$ is the resolvent of $M_{1}$ defined by $J_{\lambda}^{M_{1}}:=\left(I+\lambda M_{1}\right)^{-1}$. Furthermore, they obtained both weak and strong convergence of the proposed algorithm. Motivated by the work of Byrne et al. [8], Kazmi and Rizvi [25] introduced the following iterative algorithm for approximating a solution of SCNPP (1.5)-(1.6) which is also a fixed point of a nonexpansive mapping $S$ in real Hilbert spaces: For a given $x_{1} \in H_{1}$, let the sequences $\left\{u_{n}\right\}$ and $\left\{x_{n}\right\}$ be generated by

$$
\left\{\begin{array}{l}
u_{n}=J_{\lambda}^{M_{1}}\left(x_{n}+\gamma A^{*}\left(J_{\lambda}^{M_{2}}-I\right) A x_{n}\right), \\
x_{n+1}=\alpha_{n} f\left(x_{n}\right)+\left(1-\alpha_{n}\right) S u_{n}, \quad n \geq 1,
\end{array}\right.
$$

where $f$ is a contraction mapping on $H_{1}$. Using (1.7), they proved that both $\left\{u_{n}\right\}$ and $\left\{x_{n}\right\}$ converge strongly to $z \in F(S) \cap \Gamma$, where $\Gamma$ is the solution set of SCNPP (1.5)-(1.6).

In 2011, Moudafi [29] introduced and studied the following type of split problem, called the split variational inclusion problem (SVIP), which naturally extends the SCNPP and the VIP (1.1): Find

$$
x^{*} \in H_{1} \text { such that } 0 \in M_{1}\left(x^{*}\right)+N_{1}\left(x^{*}\right) \text {, }
$$

and such that $y^{*}=A x^{*} \in H_{2}$ solves

$$
0 \in M_{2}\left(y^{*}\right)+N_{2}\left(y^{*}\right)
$$

where $M_{1}: H_{1} \rightarrow 2^{H_{1}}$ and $M_{2}: H_{2} \rightarrow 2^{H_{2}}$ are multivalued mappings, $A$ is a bounded linear operator from $H_{1}$ to $H_{2}, N_{1}: H_{1} \rightarrow H_{1}$ and $N_{2}: H_{2} \rightarrow H_{2}$ are single-valued operators. Note that if (1.8) and (1.9) are considered separately, then (1.8) is a VIP with its solution set $\left(M_{1}+N_{1}\right)^{-1}(0)$ and (1.9) is another VIP with its solution set $\left(M_{2}+N_{2}\right)^{-1}(0)$. In [29], Moudafi proved that $x^{*} \in\left(M_{1}+N_{1}\right)^{-1}(0)$ if and only if $x^{*}=J_{\lambda}^{M_{1}}\left(I-\lambda N_{1}\right)\left(x^{*}\right), \forall \lambda>0$. It was also shown in [29] that, if $N_{1}$ is an $\alpha$-inverse strongly monotone mapping and $M_{1}$ is a maximal monotone mapping, then $J_{\lambda}^{M_{1}}(I-\lambda f)$ is averaged with $0<\lambda<2 \alpha$. Hence, $J_{\lambda}^{M_{1}}(I-\lambda f)$ is a nonexpansive mapping with $0<\lambda<2 \alpha$. Also, Moudafi [29] proposed the following iterative algorithm and obtained its weak convergence to a solution of problem (1.8)-(1.9): For $x_{1} \in H_{1}$, the sequence $\left\{x_{n}\right\}$ is generated by

$$
x_{n+1}=J_{\lambda}^{M_{1}}\left(I-\lambda N_{1}\right)\left(x_{n}+\gamma A^{*}\left(J_{\lambda}^{M_{2}}\left(I-\lambda N_{2}\right)-I\right) A x_{n}\right), \quad n \in \mathbb{N},
$$

where $\gamma \in\left(0, \frac{1}{L}\right)$ with $L$ being the spectral radius of the operator $A^{*} A$. Based on the work of Moudafi [29], Kazmi and Rizvi [25], Shehu and Ogbuisi [35] proposed the following iterative algorithm for approximating a solution of SVIP (1.8)-(1.9), which is also a fixed point of a nonexpansive mapping $S$ : For $x_{1} \in H_{1}$, let the sequence $\left\{x_{n}\right\}$ be generated by

$$
\left\{\begin{array}{l}
w_{n}=\left(1-\alpha_{n}\right) x_{n}, \\
y_{n}=J_{\lambda}^{M_{1}}\left(I-\lambda N_{1}\right)\left(w_{n}+\gamma A^{*}\left(J_{\lambda}^{M_{2}}\left(I-\lambda N_{2}\right)-I\right) A w_{n}\right), \\
x_{n+1}=\left(1-\beta_{n}\right) y_{n}+\beta_{n} S y_{n}, \quad \forall n \geq 1 .
\end{array}\right.
$$

As observed by Moudafi, the SVIP can be viewed as an important generalization of the split fixed point problem, split variational inequality problem and the SFP (see [32, 40-42]). 
Furthermore, SVIPs are generally known to be very useful in the study of wide classes of problems, especially problems arising from mechanics, optimization, nonlinear programming, economics, finance, applied sciences, among others. For more results on SVIP see $[15,22-24,34]$ and the references therein.

Recently, Takahashi and Yao [38] studied the SCNPP in a more general setting, that is, when $A$ is a bounded linear operator from a Hilbert space to a Banach space. In fact, they established the following result.

Theorem 1.1 Let $H$ be a real Hilbert space and $E$ be a uniformly convex and uniformly smooth Banach space. Let $M_{1}: H \rightarrow 2^{H}$ and $M_{2}: E \rightarrow 2^{E^{*}}$ be two multivalued maximal monotone mappings such that $\Omega:=M_{1}^{-1}(0) \cap A^{-1}\left(M_{2}^{-1}(0)\right) \neq \emptyset$. Let $A: H \rightarrow$ E be a bounded linear operator such that $A \neq 0$ and $A^{*}: E^{*} \rightarrow H$ be the adjoint of the operator $A$. Let $x_{1} \in H$ be arbitrary and the sequence $\left\{x_{n}\right\}$ be defined iteratively by

$$
\left\{\begin{array}{l}
z_{n}=J_{\lambda_{n}}^{M_{1}}\left(x_{n}-\lambda_{n} A^{*} J_{E}\left(A x_{n}-T_{\mu_{n}}^{M_{2}} A x_{n}\right)\right), \\
y_{n}=\alpha_{n} x_{n}+\left(1-\alpha_{n}\right) z_{n}, \\
C_{n}=\left\{z \in H:\left\|y_{n}-z\right\| \leq\left\|x_{n}-z\right\|\right\}, \\
Q_{n}=\left\{z \in H:\left\langle x_{n}-z, x_{1}-x_{n}\right\rangle \geq 0\right\}, \\
x_{n+1}=P_{C_{n} \cap Q_{n}} x_{1}, \quad n \geq 1,
\end{array}\right.
$$

where $\left\{\alpha_{n}\right\}$ is a sequence in $[0,1],\left\{\lambda_{n}\right\}$ and $\left\{\mu_{n}\right\}$ are sequences in $(0, \infty)$ such that $0 \leq \alpha_{n} \leq$ $a<1,0<b \leq \mu_{n}$ and $0<c \leq \lambda_{n}\|A\|^{2} \leq d<2$ for some $a, b, c, d \in \mathbb{R}$. Then $\left\{x_{n}\right\}$ converges strongly to a point $z_{0} \in \Omega$, where $z_{0}=P_{\Omega} x_{1}$.

On the other hand, the approximation of fixed points of multivalued mappings with respect to Hausdorff metric has been an area of great research interest due to its numerous applications in diverse areas such as game theory, mathematical economics, non-smooth differential equations and others. Thus, it has continued to attract the interest of numerous researchers (see, for example [4, 19, 30]). Recently, Chidume and Ezeora [13] introduced and studied a Krasnoselskii-type algorithm, and proved its strong convergence to a common fixed point of a finite family of multivalued strictly pseudocontractive mappings in a real Hilbert space. More precisely, they proved the following result.

Theorem 1.2 Let $C$ be a nonempty, closed and convex subset of a real Hilbert space $H$ and $S_{i}: C \rightarrow C B(C)$ be a finite family of multivalued $\theta_{i}$-strictly pseudocontractive mappings, $\theta_{i} \in(0,1), i=1,2, \ldots, m$ such that $\bigcap_{i=1}^{m} F\left(S_{i}\right) \neq \emptyset$. Assume that, for $p \in \bigcap_{i=1}^{m} F\left(S_{i}\right), S_{i} p=\{p\}$ and $S_{i}, i=1,2, \ldots, m$ is hemicompact and continuous. Let $\left\{x_{n}\right\}$ be a sequence defined for $x_{0} \in C$, by

$$
x_{n+1}=\alpha_{0} x_{n}+\alpha_{1} y_{n}^{1}+\alpha_{2} y_{n}^{2}+\cdots+\alpha_{m} y_{n}^{m},
$$

where $y_{n}^{i} \in S_{i} x_{n}, n \geq 1$ and $\alpha_{i} \in(\theta, 1), i=0,1, \ldots, m$ such that $\sum_{i=1}^{m} \alpha_{i}=1$ with $\theta:=$ $\max \left\{\theta_{i}, i=1, \ldots, m\right\}$. Then the sequence $\left\{x_{n}\right\}$ converges strongly to an element of $\bigcap_{i=1}^{m} F\left(S_{i}\right)$.

In the course of proving Theorem 1.2, Chidume and Ezeora [13] considered the following lemma (see also [14]). 
Lemma 1.3 Let $H$ be a real Hilbert space and $\left\{x_{i}, i=1,2, \ldots, m\right\} \subset H$. For $\alpha_{i} \in(0,1), i=$ $1,2, \ldots, m$ such that $\sum_{i=1}^{m} \alpha_{i}=1$, the following identity holds:

$$
\left\|\sum_{i=1}^{m} \alpha_{i} x_{i}\right\|^{2}=\sum_{i=1}^{m} \alpha_{i}\left\|x_{i}\right\|^{2}-\sum_{i, j=1, i \neq j}^{m} \alpha_{i} \alpha_{j}\left\|x_{i}-x_{j}\right\|^{2} .
$$

We now make the following observations about Lemma 1.3 and Algorithm (1.12).

Remark 1.4 Lemma 1.3 was established under the assumption that $\sum_{i=1}^{m} \alpha_{i}=1$, and since then many authors have used this assumption for the study of finite family of nonlinear problems (see $[11,36,37]$ and the references therein). However, we observe that in a more general case where $\left\{\alpha_{n}^{i}\right\}$ is not necessarily a constant sequence, it may be difficult to construct such sequences $\left\{\alpha_{n}^{i}\right\}, i=1,2,3, \ldots, m$ whose sum is 1 for some large $m$. Therefore, it is of practical computational importance to consider the following question.

Question Can we obtain a similar result without this assumption?

Our interest, among others, is to answer this question in the affirmative.

\section{Remark 1.5}

(i) The choice of the stepsize, $0<c \leq \lambda_{n}\|A\|^{2} \leq d<2$ used in Theorem 1.1 and some other corresponding results, requires the computation of the norm of $A$ which in general is a very difficult task to accomplish as shown in the following result.

Theorem 1.6 ([16, Theorem 2.3]) Let $p \in[1, \infty)$ be a rational number except for $p=1,2$. Unless $P=N P$, there is no algorithm which computes the $p$-norm of a matrix with entries in $\{-1,0,1\}$ to relative error with running time polynomial in the dimensions.

(ii) Algorithm (1.12) requires at each step of the iteration process, the computation of two subsets $C_{n}$ and $Q_{n}$, the computation of their intersection $C_{n} \cap Q_{n}$ and the computation of the projection of the initial starting point onto this intersection; thus, leading to an increase in the computational cost of the iteration. Hence, algorithms that do not involve the construction of $C_{n}$ and $Q_{n}$ are more interesting and of practical computational importance since they are easy to compute than those that involve these constructions. The desire to search for these algorithms, impels, urges and motivates us to undertake this research. The work of Kazmi and Rizvi [25], Moudafi [29], Shehu and Ogbuisi [35], Takahashi and Yao [38], Chidume and Ezeora [13] provides similar stimulus.

Our purposes in this paper are highlighted in the following approach: First, to study an extension of the SCNPP (1.5)-(1.6) to the following finite family of split problem: Find $x^{*} \in \bigcap_{k=1}^{K} F\left(S^{k}\right)$ such that

$$
0 \in M^{j}\left(x^{*}\right)+N^{j}\left(x^{*}\right), \quad j=1,2, \ldots, J
$$

and

$$
y^{*}=A^{i} x^{*} \in E \text { such that } 0 \in Q^{i}\left(y^{*}\right), \quad i=1,2, \ldots, I,
$$


where $H$ is a real Hilbert space, $E$ is uniformly convex and uniformly smooth real Banach space, $S^{k}: H \rightarrow P(H), k=1,2, \ldots, K$ is a finite family of type-one demicontractive mappings, $M^{j}: H \rightarrow 2^{H}, j=1,2, \ldots, J$ and $M^{i}: E \rightarrow 2^{E^{*}}, i=1,2, \ldots, I$ are two finite families of multivalued maximal monotone mappings, $N^{j}: H \rightarrow H$ is a finite family of $\alpha$-inverse strongly monotone mappings and $A^{i}$ (for each $i=1,2, \ldots, I$ ) are bounded linear operators from $H$ to $E$. Second, we propose a new viscosity-type iteration process that does not involve the construction of either $C_{n}$ or $Q_{n}$ (or both). Also, the choice of the stepsize adopted for our computation does not require prior knowledge of the operator norm. Furthermore, our control sequences do not require the imposition that their sum equals unity. Third, using our proposed algorithm, we state and prove strong convergence theorem for approximating a solution of problem (1.15)-(1.16). Fourth, we apply our results to study a finite family of split convex minimization problems, and finally consider a numerical experiment of our results to further illustrate their applicability. Our results extend and improve the results of Byrne et al. [8], Kazmi and Rizvi [25], Moudafi [29], Shehu and Ogbuisi [35], Takahashi and Yao [38], Chidume and Ezeora [13], and a host of other important results in this direction.

\section{Preliminaries}

In this section, we recall some useful definitions and results that are needed in the proof of the main results. Also, we shall denote the real Hilbert space by $H$, weak and strong convergence by $\rightarrow$ and $\rightarrow$, respectively.

Let $(X, d)$ be a metric space, $2^{X}$ be the family of all nonempty subsets of $X$ and $C B(X)$ be the family of all nonempty, closed and bounded subsets of $X$. Let $\mathcal{H}$ denote the Hausdorff metric induced by the metric $d$, that is, for all $A, B \in C B(X)$,

$$
\mathcal{H}(A, B)=\max \left\{\sup _{a \in A} d(a, B), \sup _{b \in B} d(b, A)\right\}
$$

where $d(a, B):=\inf _{b \in B} d(a, b)$.

A subset $K$ of $X$ is called proximinal if for each $x \in X$, there exists $k \in K$ such that

$$
\|x-k\|=\inf \{\|x-y\|: y \in K\}=d(x, K) .
$$

It is well known that every closed convex subset of a uniformly convex Banach space is proximinal. We shall denote the family of all proximinal subsets of $X$ by $P(X)$, for a nonempty set $X$.

Let $S: H \rightarrow 2^{H}$ be a multivalued mapping, a point $x \in H$ is called a fixed point of $S$ if $x \in S x$. If $S x=\{x\}$, then $x$ is called a strict fixed point of $S$. Throughout this paper, we shall denote the set of fixed points of $S$ by $F(S)$.

A multivalued mapping $S$ is said to be nonexpansive, if

$$
\mathcal{H}(S x, S y) \leq\|x-y\| \quad \forall x, y \in H
$$

and quasi-nonexpansive, if $F(S) \neq \emptyset$ and for all $p \in F(S)$,

$$
\mathcal{H}(S x, S p) \leq\|x-p\|
$$


Clearly, every nonexpansive mapping with nonempty fixed point set is quasi-nonexpansive.

$S$ is called $\theta$-strictly pseudocontractive in the sense of Isiogugu [17], if there exists $\theta \in$ $[0,1)$ such that $\forall x, y \in H$ and $u \in S x$ there exists $v \in S y$ satisfying $\|u-v\| \leq \mathcal{H}(S x, S y)$ and

$$
\mathcal{H}^{2}(S x, S y) \leq\|x-y\|^{2}+\theta\|x-u-(y-v)\|^{2},
$$

and demicontractive in the sense of Isiogugu and Osilike [19], if $F(S) \neq \emptyset$ and for all $p \in$ $F(S), x \in H$ there exists $\theta \in[0,1)$ such that

$$
\mathcal{H}^{2}(S x, S p) \leq\|x-p\|^{2}+\theta d^{2}(x, S x)
$$

Every nonexpansive mapping is 0 -strictly pseudocontractive mapping and every quasinonexpansive mapping is a demicontractive mapping with $\theta=0$.

The multivalued mapping $S$ is said to be of type-one in the sense of Isiogugu et al. [21] (see also [20]), if given any pair $x, y \in H$, then

$$
\|u-v\| \leq \mathcal{H}(S x, S y), \quad \text { for all } u \in P_{S} x, v \in P_{S} y
$$

where $P_{S} x:=\{u \in S x:\|u-x\|=d(x, S x)\}$.

A single-valued mapping $f: H \rightarrow H$ is called contraction, if there exists $\rho \in(0,1)$ such that

$$
\|f x-f y\| \leq \rho\|x-y\| \quad \forall x, y \in H .
$$

If $\rho=1$, then $f$ is called nonexpansive. Furthermore, if $f_{n}: H \rightarrow H$ is a uniformly convergent sequence of contractions, then there exists a sequence of real numbers $\rho_{n} \in(0,1)$ such that

$$
\left\|f_{n}(x)-f_{n}(y)\right\| \leq \rho_{n}\|x-y\|, \quad \forall x, y \in H \text { and } n \geq 1 .
$$

A mapping $f: H \rightarrow H$ is said to be

(i) Lipschitz, if there exists a constant $L>0$ such that

$$
\|f x-f y\| \leq L\|x-y\|, \quad \forall x, y \in H,
$$

(ii) monotone, if

$$
\langle f x-f y, x-y\rangle \geq 0, \quad \forall x, y \in H,
$$

(iii) $\alpha$-inverse strongly monotone, if there exists a constant $\alpha>0$ such that

$$
\langle f x-f y, x-y\rangle \geq \alpha\|f x-f y\|^{2}, \quad \forall x, y \in H .
$$

If $\alpha=1$, then $f$ is called firmly nonexpansive. Moreover, if $f$ is $\alpha$-inverse strongly monotone, then it is $\frac{1}{\alpha}$-Lipschitz continuous. 
Let $E$ be a real Banach space with $\operatorname{dim} E \geq 2$, then the modulus of convexity of $E$ denoted by $\delta_{E}:[0,2] \rightarrow[0,1]$ is defined by

$$
\delta_{E}(\epsilon):=\inf \left\{1-\left\|\frac{x+y}{2}\right\|:\|x\|=\|y\|=1, \epsilon=\|x-y\|\right\} .
$$

We say that $E$ is uniformly convex if $\delta_{E}(\epsilon)>0$, for any $\epsilon \in(0,2]$ and $p$-uniformly convex, if there exists $c_{p}>0$ such that $\delta_{E}(\epsilon) \geq c_{p} \epsilon^{p}$ for any $\epsilon \in(0,2]$.

The modulus of smoothness of $E$ denoted by $\rho_{E}(\tau):[0, \infty) \rightarrow[0, \infty)$ is defined by

$$
\rho_{E}(\tau):=\left\{\frac{\|x+\tau y\|+\|x-\tau y\|}{2}-1:\|x\|=\|y\|=1\right\} .
$$

If $\lim _{n \rightarrow \infty}\left(\frac{\rho_{E}(\tau)}{\tau}\right)=0$, then we say that $E$ is uniformly smooth. Also, if there exists $c_{q}>0$ such that $\rho_{E}(\tau) \leq c_{q} \tau^{q}$ for any $\tau>0$, then we say that $E$ is $q$-uniformly smooth. It is generally known that $p$-uniformly convex Banach spaces are uniformly convex while $q$-uniformly smooth Banach spaces are uniformly smooth. Moreover, $E$ is uniformly smoooth if and only if its dual space $E^{*}$ is unformly convex. Examples of uniformly smooth Banach spaces includes Hilbert spaces, $L_{p}$ (or $l_{p}$ ) spaces, $1<p<\infty$, and the Sobolev spaces $W_{m}^{p}, 1<p<\infty$ (see [12]). Also, it is well known (see [43]) that

$$
L_{p}\left(l_{p}\right) \text { or } W_{m}^{p} \text { is }\left\{\begin{array}{l}
p \text {-uniformly convex, if } p \geq 2, \\
2 \text {-uniformly convex, if } 1<p \leq 2 .
\end{array}\right.
$$

The normalized duality mapping $J_{E}: E \rightarrow 2^{E^{*}}$ is defined by

$$
J_{E}(x)=\left\{x^{*} \in E^{*}:\left\langle x, x^{*}\right\rangle=\|x\|^{2},\|x\|=\left\|x^{*}\right\|\right\}, \quad \forall x \in E .
$$

If $E$ is a Hilbert space, then $J_{E}=I$, where $I$ is the identity mapping. Also, if $E$ is a real uniformly smooth and uniformly convex Banach space, then $J_{E}$ and $J^{-1}: E^{*} \rightarrow E$ are single valued. Moreover, $J_{E} J^{-1}=I_{E^{*}}$ and $J^{-1} J_{E}=I_{E}$ (see [12]).

A multivalued mapping $M: E \rightarrow 2^{E^{*}}$ is called monotone, if

$$
\left\langle x-y, u^{*}-v^{*}\right\rangle \geq 0 \quad \forall x, y \in D(M), u^{*} \in M(x), v^{*} \in M(y),
$$

where $D(M)$ is the domain of $M . M$ is called maximal monotone if the graph $G(M)$ of $M$ defined by

$$
G(M)=:\left\{\left(x, u^{*}\right) \in E \times E^{*}: u^{*} \in M(x)\right\}
$$

is not properly contained in the graph of any other monotone mapping. It is known that if $M$ is a maximal monotone operator, then $R\left(I+\lambda J^{-1} M\right)=E$, where $R\left(I+\lambda J^{-1} M\right)$ is the range of $\left(I+\lambda J^{-1} M\right)$ (see [33]). Also, if $E$ is uniformly convex and smooth, then $M$ is maximal monotone if and only if $R(J+\lambda M)=E^{*}$ for $\lambda>0$ (see [5]). Hence, $R\left(I+\lambda J^{-1} M\right)=E$.

For a maximal monotone mapping $M$, the metric resolvent $T_{\lambda}^{M}: R\left(I+\lambda J^{-1} M\right)=E \rightarrow$ $D(M)$ of $M$ is defined by $T_{\lambda}^{M} x=\left(I+\lambda J^{-1} M\right)^{-1} x, \forall \lambda>0, x \in E$. It is known that $T_{\lambda}^{M}$ is single 
valued and nonexpansive. Moreover, $0 \in M x$ if and only if $x=T_{\lambda}^{M} x$ (see [31]). Furthermore, $T_{\lambda}^{M}$ satisfies the following property (see [3, p. 4]):

$$
\left\langle T_{\lambda}^{M} x-q, J\left(x-T_{\lambda}^{M} x\right)\right| \geq 0 \quad \forall x \in E, q \in F\left(T_{\lambda}^{M}\right) .
$$

If $E$ is a real Hilbert space, then the metric resolvent $T_{\lambda}^{M}$ of $M$ is simply called the resolvent of $M$ and it is defined by

$$
T_{\lambda}^{M}(x)=(I+\lambda M)^{-1} x, \quad x \in H, \lambda>0
$$

For the rest of this paper, we shall denote the metric resolvent of $M$ by $T_{\lambda}^{M}$ and the resolvent of $M$ by $J_{\lambda}^{M}$ for all $\lambda>0$.

Definition 2.1 A multivalued mapping $S: H \rightarrow P(H)$ is said to be demiclosed at the origin, if for any sequence $\left\{x_{n}\right\} \subset H$ with $x_{n} \rightarrow x$ and $d\left(x_{n}, S x_{n}\right) \rightarrow 0, n \rightarrow \infty$, we have $x \in S x$.

Lemma 2.2 ([44]) Let $C$ be a nonempty, closed and convex subset of a real uniformly smooth Banach space $E$ and $S: C \rightarrow C$ be a nonexpansive mapping. If $x_{n} \rightarrow x \in C$ and $\left\|x_{n}-S x_{n}\right\| \rightarrow 0$, then $x=S x$.

Lemma 2.3 ([12]) Let $H$ be a real Hilbert space, then, for all $x, y \in H$ and $\alpha \in(0,1)$, the following hold:

(i) $2\langle x, y\rangle=\|x\|^{2}+\|y\|^{2}-\|x-y\|^{2}=\|x+y\|^{2}-\|x\|^{2}-\|y\|^{2}$,

(ii) $\|\alpha x+(1-\alpha) y\|^{2}=\alpha\|x\|^{2}+(1-\alpha)\|y\|^{2}-\alpha(1-\alpha)\|x-y\|^{2}$,

(iii) $\|x+y\|^{2} \leq\|x\|^{2}+2\langle y, x+y\rangle$.

Lemma 2.4 ([39]) Let $\left\{a_{n}\right\}$ be a sequence of non-negative real numbers such that

$$
a_{n+1} \leq\left(1-t_{n}\right) a_{n}+t_{n} \sigma_{n}+\delta_{n}, \quad n \geq 0,
$$

where

(i) $\left\{t_{n}\right\} \subset[0,1], \sum_{n=0}^{\infty} t_{n}=\infty$,

(ii) $\lim \sup \sigma_{n} \leq 0$,

(iii) $\delta_{n} \geq 0, \sum_{n=0}^{\infty} \delta_{n}<\infty$.

Then $a_{n} \rightarrow 0$ as $n \rightarrow \infty$.

Lemma 2.5 ([27]) Let $\left\{\Gamma_{n}\right\}$ be a sequence of real numbers that does not decrease at infinity, in the sense that there exists a subsequence $\left\{\Gamma_{n_{j}}\right\}_{j \geq 0}$ of $\left\{\Gamma_{n}\right\}$ such that

$$
\Gamma_{n_{j}}<\Gamma_{n_{j}+1} \quad \forall j \geq 0 .
$$

Also consider the sequence of integers $\{\tau(n)\}_{n \geq n_{0}}$ defined by

$$
\tau(n)=\max \left\{k \leq n \mid \Gamma_{k}<\Gamma_{k+1}\right\} .
$$

Then $\left\{\Gamma_{n}\right\}_{n \geq n_{0}}$ is a nondecreasing sequence such that $\tau(n) \rightarrow \infty$, as $n \rightarrow 0$, and for all $n \geq n_{0}$, the following two estimates hold:

$$
\Gamma_{\tau(n)} \leq \Gamma_{\tau(n)+1}, \quad \Gamma_{n} \leq \Gamma_{\tau(n)+1} .
$$


Lemma 2.6 ([23] (see also [22])) Let $H$ be a real Hilbert space. Let $M: H \rightarrow 2^{H}$ be a monotone mapping and $N: H \rightarrow H$ be any nonlinear mapping. Suppose that $z=J_{\lambda}^{M}(I-\lambda N) x$ and $w=J_{\mu}^{M}(I-\mu N) x$, for $0<\lambda \leq \mu$, then

$$
\|z-x\| \leq 2\|w-x\| \quad \forall x \in H .
$$

Let $K$ be a nonempty closed and convex subset of a real Hilbert space $H$. Suppose that $\left\{T_{i}\right\}_{i=1}^{N}, N \geq 2$, is a countable finite family of mappings $T_{i}: K \rightarrow K$, in [18] the authors consider the horizontal iteration process generated from an arbitrary $x_{1}$ for the finite family of mappings $\left\{T^{i}\right\}_{i=1}^{N}$, using a finite family of the control sequences $\left\{\left\{\alpha_{n}^{i}\right\}_{n=1}^{\infty}\right\}_{i=1}^{N}$ as follows.

For $N=2$,

$$
x_{n+1}=\alpha_{n}^{1} x_{n}+\left(1-\alpha_{n}^{1}\right)\left[\alpha_{n}^{2} T_{1} x_{n}+\left(1-\alpha_{n}^{2}\right) T_{2} x_{n}\right] .
$$

For $N=3$,

$$
x_{n+1}=\alpha_{n}^{1} x_{n}+\left(1-\alpha_{n}^{1}\right)\left[\alpha_{n}^{2} T_{1} x_{n}+\left(1-\alpha_{n}^{2}\right)\left[\alpha_{n}^{3} T_{2} x_{n}+\left(1-\alpha_{n}^{3}\right) T_{3} x_{n}\right]\right] .
$$

For an arbitrary but finite $N \geq 2$,

$$
\begin{aligned}
x_{n+1}= & \alpha_{n}^{1} x_{n}+\left(1-\alpha_{n}^{1}\right)\left[\alpha_{n}^{2} T_{1} x_{n}\right. \\
& \left.+\left(1-\alpha_{n}^{2}\right)\left[\alpha_{n}^{3} T_{2} x_{n}+\left(1-\alpha_{n}^{3}\right)\left[\cdots\left[\alpha_{N} T_{N-1} x_{n}+\left(1-\alpha_{N}\right) T_{N} x_{n}\right] \cdots\right]\right]\right] \\
= & \alpha_{n}^{1} x_{n}+\sum_{i=2}^{N} \alpha_{n}^{i} \prod_{j=1}^{i-1}\left(1-\alpha_{n}^{j}\right) T_{i-1} x_{n}+\prod_{j=1}^{N}\left(1-\alpha_{n}^{j}\right) T_{N} x_{n}, \quad n \geq 1 .
\end{aligned}
$$

The proofs of the following lemmas (Lemmas 2.7 and 2.10) are given in [18]. However, we reproduce the proofs here for avoidance of doubt.

Lemma 2.7 Let $\left\{\alpha_{i}\right\}_{i=1}^{N}$ be a countable subset of the set of real numbers $\mathbb{R}$, where $N \geq 2$ is an arbitrary integer. Then the following holds:

$$
\alpha_{1}+\sum_{i=2}^{N} \alpha_{i} \prod_{j=1}^{i-1}\left(1-\alpha_{j}\right)+\prod_{j=1}^{N}\left(1-\alpha_{j}\right)=1
$$

Proof For $N=2$,

$$
\begin{aligned}
\alpha_{1}+\sum_{i=2}^{2} \alpha_{i} \prod_{j=1}^{i-1}\left(1-\alpha_{j}\right)+\prod_{j=1}^{2}\left(1-\alpha_{j}\right) & =\alpha_{1}+\alpha_{2}\left(1-\alpha_{1}\right)+\left(1-\alpha_{1}\right)\left(1-\alpha_{2}\right) \\
& =\alpha_{1}+\left(1-\alpha_{1}\right)\left[\alpha_{2}+\left(1-\alpha_{2}\right)\right] \\
& =\alpha_{1}+\left(1-\alpha_{1}\right)=1 .
\end{aligned}
$$


We assume it is true for $N$ and prove for $N+1$.

$$
\begin{aligned}
& \alpha_{1}+\sum_{i=2}^{N+1} \alpha_{i} \prod_{j=1}^{i-1}\left(1-\alpha_{j}\right)+\prod_{j=1}^{N+1}\left(1-\alpha_{j}\right) \\
& =\alpha_{1}+\sum_{i=2}^{N} \alpha_{i} \prod_{j=1}^{i-1}\left(1-\alpha_{j}\right)+\alpha_{N+1} \prod_{j=1}^{N}\left(1-\alpha_{j}\right)+\prod_{j=1}^{N+1}\left(1-\alpha_{j}\right) \\
& =\alpha_{1}+\sum_{i=2}^{N} \alpha_{i} \prod_{j=1}^{i-1}\left(1-\alpha_{j}\right)+\prod_{j=1}^{N}\left(1-\alpha_{j}\right)\left[\alpha_{N+1}+\left(1-\alpha_{N+1}\right)\right] \\
& =\alpha_{1}+\sum_{i=2}^{N} \alpha_{i} \prod_{j=1}^{i-1}\left(1-\alpha_{j}\right)+\prod_{j=1}^{N}\left(1-\alpha_{j}\right) \\
& =1 .
\end{aligned}
$$

Remark 2.8 Lemma 2.7 holds if $\left\{\alpha_{i}\right\}_{i=1}^{N}$ is replaced with $\left\{\alpha_{i}\right\}_{i=0}^{N}$, and $N \geq 2$ is replaced with $N \geq 1$.

Lemma 2.9 Let $\left\{\alpha_{i}\right\}_{i=k}^{N}$ be a countable subset of the set of real numbers $\mathbb{R}$, where $k$ is a fixed non-negative integer and $N \in \mathbb{N}$ is any integer with $k+1 \leq N$. Then the following holds:

$$
\alpha_{k}+\sum_{i=k+1}^{N} \alpha_{i} \prod_{j=k}^{i-1}\left(1-\alpha_{j}\right)+\prod_{j=k}^{N}\left(1-\alpha_{j}\right)=1 .
$$

Proof For $k=0$ and $k=1$, the proofs follow from Remark 2.8 and Lemma 2.7, respectively.

We assume it is true for $k$ and $N$. Now for $k$ and $N+1$,

$$
\begin{aligned}
& \alpha_{k}+\sum_{i=k+1}^{N+1} \alpha_{i} \prod_{j=k}^{i-1}\left(1-\alpha_{j}\right)+\prod_{j=k}^{N+1}\left(1-\alpha_{j}\right) \\
& =\alpha_{k}+\sum_{i=k+1}^{N} \alpha_{i} \prod_{j=k}^{i-1}\left(1-\alpha_{j}\right)+\alpha_{N+1} \prod_{j=k}^{N}\left(1-\alpha_{j}\right)+\prod_{j=k}^{N+1}\left(1-\alpha_{j}\right) \\
& =\alpha_{k}+\sum_{i=k+1}^{N} \alpha_{i} \prod_{j=k}^{i-1}\left(1-\alpha_{j}\right)+\prod_{j=k}^{N}\left(1-\alpha_{j}\right)\left[\alpha_{N+1}+\left(1-\alpha_{N+1}\right)\right] \\
& =\alpha_{k}+\sum_{i=k+1}^{N} \alpha_{i} \prod_{j=k}^{i-1}\left(1-\alpha_{j}\right)+\prod_{j=k}^{N}\left(1-\alpha_{j}\right) \\
& =\alpha_{k}+\sum_{i=k}^{N} \alpha_{i} \prod_{j=k}^{i-1}\left(1-\alpha_{j}\right)+\prod_{j=k}^{N}\left(1-\alpha_{j}\right)=1 .
\end{aligned}
$$

Lemma 2.10 Let $t, u$ and $v$ be arbitrary elements of a real Hilbert space H. Let $k$ be a fixed non-negative integer and $N \in \mathbb{N}$ be such that $k+1 \leq N$. Let $\left\{v_{i}\right\}_{i=k}^{N-1} \subseteq H$ and $\left\{\alpha_{i}\right\}_{i=k}^{N} \subseteq[0,1]$ be a countable finite subset of $H$ and $\mathbb{R}$, respectively. Define

$$
y=\alpha_{k} t+\sum_{i=k+1}^{N} \alpha_{i} \prod_{j=k}^{i-1}\left(1-\alpha_{j}\right) v_{i-1}+\prod_{j=k}^{N}\left(1-\alpha_{j}\right) v .
$$


Then

$$
\begin{aligned}
\|y-u\|^{2}= & \alpha_{k}\|t-u\|^{2}+\sum_{i=k+1}^{N} \alpha_{i} \prod_{j=k}^{i-1}\left(1-\alpha_{j}\right)\left\|v_{i-1}-u\right\|^{2}+\prod_{j=k}^{N}\left(1-\alpha_{j}\right)\|v-u\|^{2} \\
& -\alpha_{k}\left[\sum_{i=k+1}^{N} \alpha_{i} \prod_{j=k}^{i-1}\left(1-\alpha_{j}\right)\left\|t-v_{i-1}\right\|^{2}+\prod_{j=k}^{N}\left(1-\alpha_{j}\right)\|t-v\|^{2}\right] \\
& -\left(1-\alpha_{k}\right)\left[\sum_{i=k+1}^{N-1} \alpha_{i} \prod_{j=k}^{i}\left(1-\alpha_{j}\right)\left\|v_{i-1}-\left[\alpha_{i+1} v_{i}+w_{i+1}\right]\right\|^{2}\right. \\
& \left.+\alpha_{N} \prod_{j=k}^{N}\left(1-\alpha_{j}\right)\left\|v-v_{N-1}\right\|^{2}\right]
\end{aligned}
$$

where $w_{k}=\sum_{i=k+1}^{N} \alpha_{i} \prod_{j=k}^{i-1}\left(1-\alpha_{j}\right) v_{i-1}+\prod_{j=k}^{N}\left(1-\alpha_{j}\right) v, k=1,2, \ldots, N-1$ and $w_{N}=\left(1-\alpha_{N}\right) v$.

Proof Observe that, for $k \leq N-1, w_{k}=\left(1-\alpha_{k}\right)\left[\alpha_{k+1} v_{k}+w_{k+1}\right]$. Consequently, we obtain from Lemma 2.3(ii)

$$
\begin{aligned}
\|y-u\|^{2}= & \left\|\alpha_{k} t+\sum_{i=k+1}^{N} \alpha_{i} \prod_{j=k}^{i-1}\left(1-\alpha_{j}\right) v_{i-1}+\prod_{j=k}^{N}\left(1-\alpha_{j}\right) v-u\right\|^{2}, \\
= & \left\|\alpha_{k} t+w_{k}-u\right\|^{2} \\
= & \left\|\alpha_{k} t+\left(1-\alpha_{k}\right)\left[\alpha_{k+1} v_{k}+w_{k+1}\right]-u\right\|^{2} \\
= & \alpha_{k}\|t-u\|^{2}+\left(1-\alpha_{k}\right)\left\|\alpha_{k+1} v_{k}+w_{k+1}-u\right\|^{2} \\
& -\alpha_{k}\left(1-\alpha_{k}\right)\left\|t-\left[\alpha_{k+1} v_{k}+w_{k+1}\right]\right\|^{2} \\
= & \alpha_{k}\|t-u\|^{2}+\left(1-\alpha_{k}\right)\left[\alpha_{k+1}\left\|v_{k}-u\right\|^{2}+\left(1-\alpha_{k+1}\right)\left\|\alpha_{k+2} v_{k+1}+w_{k+2}-u\right\|^{2}\right. \\
& \left.-\alpha_{k+1}\left(1-\alpha_{k+1}\right)\left\|v_{k}-\left[\alpha_{k+2} v_{k+1}+w_{k+2}\right]\right\|^{2}\right] \\
& -\alpha_{k}\left(1-\alpha_{k}\right)\left[\alpha_{k+1}\left\|t-v_{k}\right\|^{2}+\left(1-\alpha_{k+1}\right)\left\|t-\left[\alpha_{k+2} v_{k+1}+w_{k+2}\right]\right\|^{2}\right. \\
& \left.-\alpha_{k+1}\left(1-\alpha_{k+1}\right)\left\|v_{k}-\left[\alpha_{k+2} v_{k+1}+w_{k+2}\right]\right\|^{2}\right] \\
= & \alpha_{k}\|t-u\|^{2}+\left(1-\alpha_{k}\right) \alpha_{k+1}\left\|v_{k}-u\right\|^{2} \\
& +\left(1-\alpha_{k}\right)\left(1-\alpha_{k+1}\right)\left\|\alpha_{k+2} v_{k+1}+w_{k+2}-u\right\|^{2} \\
& -\left(1-\alpha_{k}\right) \alpha_{k+1}\left(1-\alpha_{k+1}\right)\left\|v_{k}-\left[\alpha_{k+2} v_{k+1}+w_{k+2}\right]\right\|^{2} \\
& -\alpha_{k}\left(1-\alpha_{k}\right) \alpha_{k+1}\left\|t-v_{k}\right\|^{2}-\alpha_{k}\left(1-\alpha_{k}\right)\left(1-\alpha_{k+1}\right)\left\|t-\left[\alpha_{k+2} v_{k+1}+w_{k+2}\right]\right\|^{2} \\
& +\alpha_{k}\left(1-\alpha_{k}\right) \alpha_{k+1}\left(1-\alpha_{k+1}\right)\left\|v_{k}-\left[\alpha_{k+2} v_{k+1}+w_{k+2}\right]\right\|^{2} \\
= & \alpha_{k}\|t-u\|^{2}+\left(1-\alpha_{k}\right) \alpha_{k+1}\left\|v_{k}-u\right\|^{2}-\alpha_{k}\left(1-\alpha_{k}\right) \alpha_{k+1}\left\|t-v_{k}\right\|^{2} \\
& +\left(1-\alpha_{k}\right)\left(1-\alpha_{k+1}\right)\left\|\alpha_{k+2} v_{k+1}+w_{k+2}-u\right\|^{2} \\
& -\alpha_{k}\left(1-\alpha_{k}\right)\left(1-\alpha_{k+1}\right)\left\|t-\left[\alpha_{k+2} v_{k+1}+w_{k+2}\right]\right\|^{2} \\
& -\alpha_{k+1}\left(1-\alpha_{k}\right)\left(1-\alpha_{k+1}\right)\left(1-\alpha_{k}\right)\left\|v_{k}-\left[\alpha_{k+2} v_{k+1}+w_{k+2}\right]\right\|^{2} \\
= & \alpha_{k}\|t-u\|^{2}+\left(1-\alpha_{k}\right) \alpha_{k+1}\left\|v_{k}-u\right\|^{2}-\alpha_{k}\left(1-\alpha_{k}\right) \alpha_{k+1}\left\|t-v_{k}\right\|^{2} \\
&
\end{aligned}
$$




$$
\begin{aligned}
& -\alpha_{k+1}\left(1-\alpha_{k}\right)\left(1-\alpha_{k+1}\right)\left(1-\alpha_{k}\right)\left\|v_{k}-\left[\alpha_{k+2} v_{k+1}+w_{k+2}\right]\right\|^{2} \\
& +\left(1-\alpha_{k}\right)\left(1-\alpha_{k+1}\right)\left\|\alpha_{k+2} v_{k+1}+w_{k+2}-u\right\|^{2} \\
& -\alpha_{k}\left(1-\alpha_{k}\right)\left(1-\alpha_{k+1}\right)\left\|t-\left[\alpha_{k+2} v_{k+1}+w_{k+2}\right]\right\|^{2} \\
& =\alpha_{k}\|t-u\|^{2}+\left(1-\alpha_{k}\right) \alpha_{k+1}\left\|v_{k}-u\right\|^{2}-\alpha_{k}\left(1-\alpha_{k}\right) \alpha_{k+1}\left\|t-v_{k}\right\|^{2} \\
& -\alpha_{k+1}\left(1-\alpha_{k}\right)\left(1-\alpha_{k+1}\right)\left(1-\alpha_{k}\right)\left\|v_{k}-\left[\alpha_{k+2} v_{k+1}+w_{k+2}\right]\right\|^{2} \\
& +\left(1-\alpha_{k}\right)\left(1-\alpha_{k+1}\right)\left\|\alpha_{k+2} v_{k+1}+\left(1-\alpha_{k+2}\right)\left[\alpha_{k+3} v_{k+2}+w_{k+3}\right]-u\right\|^{2} \\
& -\alpha_{k}\left(1-\alpha_{k}\right)\left(1-\alpha_{k+1}\right)\left\|\alpha_{k+2} v_{k+1}+\left(1-\alpha_{k+2}\right)\left[\alpha_{k+3} v_{k+2}+w_{k+3}\right]-t\right\|^{2} \\
& =\alpha_{k}\|t-u\|^{2}+\left(1-\alpha_{n}^{1}\right) \alpha_{k+1}\left\|v_{k}-u\right\|^{2}-\alpha_{n}^{1}\left(1-\alpha_{n}^{1}\right) \alpha_{k+1}\left\|t-v_{k}\right\|^{2} \\
& +\left(1-\alpha_{k}\right)\left(1-\alpha_{k+1}\right) \alpha_{k+2}\left\|v_{k+1}-u\right\|^{2} \\
& +\left(1-\alpha_{k}\right)\left(1-\alpha_{k+1}\right)\left(1-\alpha_{k+2}\right)\left\|\alpha_{k+3} v_{k+2}+w_{k+3}-u\right\|^{2} \\
& -\left(1-\alpha_{k}\right)\left(1-\alpha_{k+1}\right) \alpha_{k+2}\left(1-\alpha_{k+2}\right)\left\|v_{k+1}-\left[\alpha_{k+3} v_{k+2}+w_{k+3}\right]\right\|^{2} \\
& -\alpha_{k}\left(1-\alpha_{k}\right)\left(1-\alpha_{k+1}\right) \alpha_{k+2}\left\|v_{k+1}-t\right\|^{2} \\
& -\alpha_{k}\left(1-\alpha_{k}\right)\left(1-\alpha_{k+1}\right)\left(1-\alpha_{k+2}\right)\left\|\alpha_{k+3} \nu_{k+2}+w_{k+3}-t\right\|^{2} \\
& +\alpha_{k}\left(1-\alpha_{k}\right)\left(1-\alpha_{k+1}\right) \alpha_{k+2}\left(1-\alpha_{k+2}\left\|v_{k+1}-\alpha_{k+3} v_{k+2}+w_{k+3}\right\|^{2}\right. \\
& -\alpha_{k+1}\left(1-\alpha_{k}\right)\left(1-\alpha_{k+1}\right)\left(1-\alpha_{k}\right)\left\|v_{k}-\left[\alpha_{k+2} v_{k+1}+w_{k+2}\right]\right\|^{2} \\
& =\alpha_{k}\|t-u\|^{2}+\left(1-\alpha_{k}\right) \alpha_{k+1}\left\|v_{k}-u\right\|^{2}-\alpha_{k}\left(1-\alpha_{k}\right) \alpha_{k+1}\left\|t-v_{k}\right\|^{2} \\
& +\left(1-\alpha_{k}\right)\left(1-\alpha_{k+1}\right) \alpha_{k+2}\left\|v_{k+1}-u\right\|^{2} \\
& +\left(1-\alpha_{k}\right)\left(1-\alpha_{k+1}\right)\left(1-\alpha_{k+2}\right)\left\|\alpha_{k+3} v_{k+2}+w_{k+3}-u\right\|^{2} \\
& -\alpha_{k}\left(1-\alpha_{k}\right)\left(1-\alpha_{k+1}\right) \alpha_{k+2}\left\|v_{k+1}-t\right\|^{2} \\
& -\alpha_{k}\left(1-\alpha_{k}\right)\left(1-\alpha_{k+1}\right)\left(1-\alpha_{k+2}\right)\left\|\alpha_{k+3} v_{k+2}+w_{k+3}-t\right\|^{2} \\
& -\alpha_{k+1}\left(1-\alpha_{k}\right)\left(1-\alpha_{k+1}\right)\left(1-\alpha_{k}\right)\left\|v_{k}-\left[\alpha_{k+2} v_{k+1}+w_{k+2}\right]\right\|^{2} \\
& -\left(1-\alpha_{k}\right)^{2}\left(1-\alpha_{k+1}\right) \alpha_{k+2}\left(1-\alpha_{k+2}\right)\left\|v_{k+1}-\left[\alpha_{k+3} v_{k+2}+w_{k+3}\right]\right\|^{2} \\
& \vdots \\
& =\alpha_{k}\|t-u\|^{2}+\sum_{i=k+1}^{k+2} \alpha_{i} \prod_{j=k}^{i-1}\left(1-\alpha_{j}\right)\left\|v_{i-1}-u\right\|^{2} \\
& -\alpha_{k}\left[\sum_{i=k+1}^{k+2} \alpha_{i} \prod_{j=k}^{i-1}\left(1-\alpha_{j}\right)\left\|t-v_{i-1}\right\|^{2}\right] \\
& -\left(1-\alpha_{k}\right)\left[\sum_{i=k+1}^{k+2} \alpha_{i} \prod_{j=k}^{i}\left(1-\alpha_{j}\right)\left\|v_{i-1}-\left[\alpha_{i+1} v_{i}+w_{i+1}\right]\right\|^{2}\right. \\
& +\prod_{j=k}^{k+2}\left(1-\alpha_{j}\right)\left\|\left[\alpha_{k+3} v_{k+2}+w_{k+3}\right]-u\right\|^{2} \\
& -\alpha_{k} \prod_{j=k}^{k+2}\left(1-\alpha_{j}\right)\left\|t-\left[\alpha_{k+3} v_{k+2}+w_{k+3}\right]\right\|^{2}
\end{aligned}
$$




$$
\begin{aligned}
= & \alpha_{k}\|t-u\|^{2}+\sum_{i=k+1}^{N} \alpha_{i} \prod_{j=k}^{i-1}\left(1-\alpha_{j}\right)\left\|v_{i-1}-u\right\|^{2}+\prod_{j=k}^{N}\left(1-\alpha_{j}\right)\|v-u\|^{2} \\
& -\alpha_{k}\left[\sum_{i=k+1}^{N} \alpha_{i} \prod_{j=k}^{i-1}\left(1-\alpha_{j}\right)\left\|t-v_{i-1}\right\|^{2}+\prod_{j=k}^{N}\left(1-\alpha_{j}\right)\|t-v\|^{2}\right] \\
& -\left(1-\alpha_{k}\right)\left[\sum_{i=k+1}^{N-1} \alpha_{i} \prod_{j=k}^{i}\left(1-\alpha_{j}\right)\left\|v_{i-1}-\left[\alpha_{i+1} v_{i}+w_{i+1}\right]\right\|^{2}\right. \\
& \left.+\alpha_{N} \prod_{j=k}^{N}\left(1-\alpha_{j}\right)\left\|v-v_{N-1}\right\|^{2}\right] .
\end{aligned}
$$

\section{Main results}

Lemma 3.1 Let $H$ be a real Hilbert space and $E$ be a real uniformly convex and uniformly smooth Banach space. Let $j=1,2, \ldots, J, i=1,2, \ldots, I, k=1,2, \ldots, K$, for some $J, I, K \in \mathbb{N}$. For each $i, j, k$, let $A^{i}: H \rightarrow E$ be a bounded linear operator and $\left(A^{i}\right)^{*}: E^{*} \rightarrow H$ be the adjoint of $A^{i}$, let $M^{j}: H \rightarrow 2^{H}$ be a multivalued maximal monotone mapping, $N^{j}: H \rightarrow H$ be an $\alpha^{j}$-inverse strongly monotone mapping, $S^{k}: H \rightarrow P(H), k=1,2, \ldots, K$, be a finite family of multivalued type-one demicontractive mappings with coefficient $\theta^{k} \in[0,1)$, and $Q^{i}: E \rightarrow$ $2^{E^{*}}$ be a multivalued maximal monotone mapping such that $\Upsilon: \bigcap_{k=1}^{K} F\left(S^{k}\right) \cap \bigcap_{j=1}^{J}\left(M^{j}+\right.$ $\left.N^{j}\right)^{-1}(0) \cap \bigcap_{i=1}^{I}\left(A^{i}\right)^{-1}\left(\left(Q^{i}\right)^{-1}(0)\right) \neq \emptyset$. Let the stepsize $\gamma_{n}^{i}$ be chosen such that, for some $\epsilon^{i}>$ $0, \gamma_{n}^{i} \in\left(\epsilon^{i}, \frac{2\left\|A^{i} x_{n}-T_{\mu^{2}}^{Q^{i}}{ }^{i} x_{n}\right\|^{2}}{\left\|\left(A^{i}\right)^{*} J_{E}\left(A^{i} x_{n}-T_{\mu^{i}}^{M^{i}} A^{i} x_{n}\right)\right\|^{2}}-\epsilon^{i}\right)$, if $T_{\mu^{i}}^{Q^{i}} A^{i} x_{n} \neq A^{i} x_{n}$; otherwise $\gamma_{n}^{i}=\gamma^{i}$ ( $\gamma^{i}$ being any nonnegative real number). Let $f_{n}: H \rightarrow H$ be a sequence of $\rho_{n}$-contractive mappings with $0<\underline{\rho} \leq \rho_{n} \leq \bar{\rho}<1$ such that $\left\{f_{n}(x)\right\}$ is uniformly convergent for any $x \in D$, where $D$ is any bounded subset of $H$. For arbitrary $x_{1} \in H$, define the sequence $\left\{x_{n}\right\}$ iterative by

$$
\left\{\begin{array}{l}
w_{n}^{i}=x_{n}-\gamma_{n}^{i}\left(A^{i}\right)^{*} J_{E}\left(A^{i} x_{n}-T_{\mu^{i}}^{Q^{i}} A^{i} x_{n}\right), \quad i=1,2, \ldots, I, \\
w_{n}=\eta_{n}^{1} x_{n}+\sum_{i=2}^{I} \eta_{n}^{i} \prod_{t=1}^{i-1}\left(1-\eta_{n}^{t}\right) w_{n}^{i-1}+\prod_{t=1}^{I}\left(1-\eta_{n}^{t}\right) w_{n}^{I}, \\
y_{n}^{j}=J_{\lambda_{n}^{j}}^{M^{j}}\left(I-\lambda_{n}^{j} N^{j}\right) w_{n}, \quad j=1,2, \ldots, J, \\
y_{n}=\alpha_{n}^{1} w_{n}+\sum_{j=2}^{J} \alpha_{n}^{j} \prod_{t=1}^{j-1}\left(1-\alpha_{n}^{t}\right) y_{n}^{j-1}+\prod_{t=1}^{J}\left(1-\alpha_{n}^{t}\right) y_{n}^{J}, \\
u_{n}=\zeta_{n}^{1} x_{n}+\sum_{k=2}^{K} \zeta_{n}^{k} \prod_{t=1}^{k-1}\left(1-\zeta_{n}^{t}\right) u_{n}^{k-1}+\prod_{t=1}^{K}\left(1-\zeta_{n}^{t}\right) u_{n}^{K}, \\
x_{n+1}=\beta_{n}^{1} f_{n}\left(x_{n}\right)+\left(1-\beta_{n}^{1}\right)\left[\beta_{n}^{2} x_{n}+\left(1-\beta_{n}^{2}\right)\left(v u_{n}+(1-v) y_{n}\right)\right],
\end{array}\right.
$$

where $u_{n}^{k} \in P_{S^{k}} x_{n}:=\left\{u_{n}^{k} \in S^{k} x_{n}:\left\|u_{n}^{k}-x_{n}\right\|=d\left(x_{n}, S^{k} x_{n}\right)\right\}, k=1,2, \ldots, K, \mu>0, v \in(0,1)$, $0<\lambda^{j} \leq \lambda_{n}^{j}<2 \alpha^{j},\left\{\beta_{n}^{1}\right\}_{n=1}^{\infty},\left\{\beta_{n}^{2}\right\}_{n=1}^{\infty},\left\{\alpha_{n}^{j}\right\}_{n=1}^{\infty},\left\{\eta_{n}^{i}\right\}_{n=1}^{\infty}$ and $\left\{\zeta_{n}^{k}\right\}_{n=1}^{\infty}$ are real sequences in $(0,1)$ such that $\zeta_{n}^{1} \geq \theta^{k} \forall n \geq 1, k=1,2, \ldots, K$.

Then the sequence $\left\{x_{n}\right\}$ is bounded.

Proof Let $q \in \Upsilon$, then, for each $i=1,2, \ldots, I$, we obtain from (3.1) and (2.6)

$$
\begin{aligned}
\left\|w_{n}^{i}-q\right\|^{2}= & \left\|x_{n}-\gamma_{n}^{i}\left(A^{i}\right)^{*} J_{E}\left(A^{i} x_{n}-T_{\mu^{i}}^{Q^{i}} A^{i} x_{n}\right)-q\right\|^{2} \\
= & \left\|x_{n}-q\right\|^{2}+\left(\gamma_{n}^{i}\right)^{2}\left\|\left(A^{i}\right)^{*} J_{E}\left(A^{i} x_{n}-T_{\mu^{i}}^{Q^{i}} A^{i} x_{n}\right)\right\|^{2} \\
& -2 \gamma_{n}\left(x_{n}-q,\left(A^{i}\right)^{*} J_{E}\left(A^{i} x_{n}-T_{\mu^{i}}^{Q^{i}} A^{i} x_{n}\right)\right\rangle
\end{aligned}
$$




$$
\begin{aligned}
= & \left\|x_{n}-q\right\|^{2}+\gamma_{n}^{2}\left\|\left(A^{i}\right)^{*} J_{E}\left(A^{i} x_{n}-T_{\mu^{i}}^{Q^{i}} A^{i} x_{n}\right)\right\|^{2} \\
& -2 \gamma_{n}\left\langle A^{i} x_{n}-A^{i} q, J_{E}\left(A^{i} x_{n}-T_{\mu^{i}}^{Q^{i}} A^{i} x_{n}\right)\right\rangle \\
= & \left\|x_{n}-q\right\|^{2}+\gamma_{n}^{2}\left\|\left(A^{i}\right)^{*} J_{E}\left(A^{i} x_{n}-T_{\mu^{i}}^{Q^{i}} A^{i} x_{n}\right)\right\|^{2} \\
& -2 \gamma_{n}\left\langle A^{i} x_{n}-T_{\mu^{i}}^{Q^{i}} A^{i} x_{n}, J_{E}\left(A^{i} x_{n}-T_{\mu^{i}}^{Q^{i}} A^{i} x_{n}\right)\right\rangle \\
& -2 \gamma_{n}\left\langle T_{\mu_{i}}^{Q^{i}} A^{i} x_{n}-T_{\mu^{i}}^{Q^{i}} A^{i} q, J_{E}\left(A^{i} x_{n}-T_{\mu^{i}}^{Q^{i}} A^{i} x_{n}\right)\right\rangle \\
\leq & \left\|x_{n}-q\right\|^{2}+\gamma_{n}^{2}\left\|\left(A^{i}\right)^{*} J_{E}\left(A_{i} x_{n}-T_{\mu^{i}}^{Q^{i}} A^{i} x_{n}\right)\right\|^{2} \\
& -2 \gamma_{n}\left\langle A^{i} x_{n}-T_{\mu^{i}}^{Q^{i}} A^{i} x_{n}, J_{E}\left(A^{i} x_{n}-T_{\mu^{i}}^{Q^{i}} A^{i} x_{n}\right)\right\rangle \\
= & \left\|x_{n}-q\right\|^{2}+\gamma_{n}^{2}\left\|\left(A^{i}\right)^{*} J_{E}\left(A^{i} x_{n}-T_{\mu^{i}}^{Q^{i}} A^{i} x_{n}\right)\right\|^{2}-2 \gamma_{n}\left\|A^{i} x_{n}-T_{\mu^{i}}^{Q^{i}} A^{i} x_{n}\right\|^{2} \\
= & \left\|x_{n}-q\right\|^{2}-\gamma_{n}\left[2\left\|A^{i} x_{n}-T_{\mu^{i}}^{Q^{i}} A^{i} x_{n}\right\|^{2}-\gamma_{n}\left\|\left(A^{i}\right)^{*} J_{E}\left(A^{i} x_{n}-T_{\mu^{i}}^{Q^{i}} A^{i} x_{n}\right)\right\|^{2}\right] \\
\leq & \left\|x_{n}-q\right\|^{2} .
\end{aligned}
$$

From (3.1), (3.2), Lemma 2.7 and Lemma 2.10 (that is, setting $k=1$ in Lemma 2.10), we obtain

$$
\begin{aligned}
\left\|y_{n}^{j}-q\right\|^{2}= & \left\|J_{\lambda_{n}^{j}}^{M^{j}}\left(I-\lambda_{n}^{j} N^{j}\right) w_{n}-J_{\lambda_{n}^{j}}^{M^{j}}\left(I-\lambda_{n}^{j} N^{j}\right) q\right\|^{2} \\
\leq & \left\|w_{n}-q\right\|^{2} \\
= & \left\|\eta_{n}^{1} x_{n}+\sum_{i=2}^{I} \eta_{n}^{i} \prod_{t=1}^{i-1}\left(1-\eta_{n}^{t}\right) w_{n}^{i-1}+\prod_{t=1}^{I}\left(1-\eta_{n}^{t}\right) w_{n}^{I}-q\right\|^{2} \\
\leq & \eta_{n}^{1}\left\|x_{n}-q\right\|^{2}+\sum_{i=2}^{I} \eta_{n}^{i} \prod_{t=1}^{i-1}\left(1-\eta_{n}^{t}\right)\left\|w_{n}^{i-1}-q\right\|^{2}+\prod_{t=1}^{I}\left(1-\eta_{n}^{t}\right)\left\|w_{n}^{I}-q\right\|^{2} \\
& -\eta_{n}^{1}\left[\sum_{i=2}^{I} \eta_{n}^{i} \prod_{t=1}^{i-1}\left(1-\eta_{n}^{t}\right)\left\|x_{n}-w_{n}^{i-1}\right\|^{2}+\prod_{t=1}^{I}\left(1-\eta_{n}^{t}\right)\left\|x_{n}-w_{n}^{I}\right\|^{2}\right] \\
\leq & \eta_{n}^{1}\left\|x_{n}-q\right\|^{2}+\sum_{i=2}^{I} \eta_{n}^{i} \prod_{t=1}^{i-1}\left(1-\eta_{n}^{t}\right)\left\|x_{n}-q\right\|^{2}+\prod_{t=1}^{I}\left(1-\eta_{n}^{t}\right)\left\|x_{n}-q\right\|^{2} \\
& -\eta_{n}^{1}\left[\sum_{i=2}^{I} \eta_{n}^{i} \prod_{t=1}^{i-1}\left(1-\eta_{n}^{t}\right)\left\|x_{n}-w_{n}^{i-1}\right\|^{2}+\prod_{t=1}^{I}\left(1-\eta_{n}^{t}\right)\left\|x_{n}-w_{n}^{I}\right\|^{2}\right] \\
\leq & \left\|x_{n}-q\right\|^{2}-\eta_{n}^{1}\left[\sum_{i=2}^{I} \eta_{n}^{i} \prod_{t=1}^{i-1}\left(1-\eta_{n}^{t}\right)\left\|x_{n}-w_{n}^{i-1}\right\|^{2}+\prod_{t=1}^{I}\left(1-\eta_{n}^{t}\right)\left\|x_{n}-w_{n}^{I}\right\|^{2}\right] \\
\leq & \left\|x_{n}-q\right\|^{2} .
\end{aligned}
$$

Let $m_{n}=v u_{n}+(1-v) y_{n}$. Then, by (3.3), (3.4), Lemmas 2.7 and 2.10, we obtain

$$
\begin{aligned}
\left\|m_{n}-q\right\|^{2} & \leq v\left\|u_{n}-q\right\|^{2}+(1-v)\left\|y_{n}-q\right\|^{2} \\
& \leq v\left\|u_{n}-q\right\|^{2}+(1-v)\left\|\alpha_{n}^{1} w_{n}+\sum_{j=2}^{J} \alpha_{n}^{j} \prod_{t=1}^{j-1}\left(1-\alpha_{n}^{t}\right) y_{n}^{j-1}+\prod_{t=1}^{J}\left(1-\alpha_{n}^{t}\right) y_{n}^{J}-q\right\|^{2}
\end{aligned}
$$




$$
\begin{aligned}
\leq & v\left\|u_{n}-q\right\|^{2}+(1-v)\left[\alpha_{n}^{1}\left\|w_{n}-q\right\|^{2}+\sum_{j=2}^{J} \alpha_{n}^{j} \prod_{t=1}^{j-1}\left(1-\alpha_{n}^{t}\right)\left\|y_{n}^{j-1}-q\right\|^{2}\right. \\
& \left.+\prod_{t=1}^{J}\left(1-\alpha_{n}^{t}\right)\left\|y_{n}^{J}-q\right\|^{2}\right] \\
& -(1-v) \alpha_{n}^{1}\left[\sum_{j=2}^{J} \alpha_{n}^{j} \prod_{t=1}^{j-1}\left(1-\alpha_{n}^{t}\right)\left\|w_{n}-y_{n}^{j-1}\right\|^{2}+\prod_{t=1}^{J}\left(1-\alpha_{n}^{t}\right)\left\|w_{n}-y_{n}^{J}\right\|^{2}\right] \\
\leq & v\left\|u_{n}-q\right\|^{2}+(1-v)\left[\alpha_{n}^{1}\left\|w_{n}-q\right\|^{2}+\sum_{j=2}^{J} \alpha_{n}^{j} \prod_{t=1}^{j-1}\left(1-\alpha_{n}^{t}\right)\left\|w_{n}-q\right\|^{2}\right. \\
& \left.+\prod_{t=1}^{J}\left(1-\alpha_{n}^{t}\right)\left\|w_{n}-q\right\|^{2}\right] \\
& -(1-v) \alpha_{n}^{1}\left[\sum_{j=2}^{J} \alpha_{n}^{j} \prod_{t=1}^{j-1}\left(1-\alpha_{n}^{t}\right)\left\|w_{n}-y_{n}^{j-1}\right\|^{2}+\prod_{t=1}^{J}\left(1-\alpha_{n}^{t}\right)\left\|w_{n}-y_{n}^{J}\right\|^{2}\right] \\
= & v\left\|u_{n}-q\right\|^{2}+(1-v)\left\|w_{n}-q\right\|^{2} \\
& -(1-v) \alpha_{n}^{1}\left[\sum_{j=2}^{J} \alpha_{n}^{j} \prod_{t=1}^{j-1}\left(1-\alpha_{n}^{t}\right)\left\|w_{n}-y_{n}^{j-1}\right\|^{2}+\prod_{t=1}^{J}\left(1-\alpha_{n}^{t}\right)\left\|w_{n}-y_{n}^{J}\right\|^{2}\right] \\
& \left.+\prod_{t=1}^{J}\left(1-\alpha_{n}^{t}\right)\left\|w_{n}-y_{n}^{J}\right\|^{2}\right] . \\
& -(1-v)\left\|_{n}^{2}+(1-v)\right\| x_{n}-q \|^{2} \\
& \left(\sum_{j=2}^{J} \alpha_{n}^{j} \prod_{t=1}^{j-1}\left(1-\alpha_{n}^{t}\right)\left\|w_{n}-y_{n}^{j-1}\right\|^{2}\right.
\end{aligned}
$$

Since $S^{k}$ is of type-one demicontractive for each $k=1,2, \ldots, K$, we obtain from (3.1), and Lemmas 2.7 and 2.10

$$
\begin{aligned}
\left\|u_{n}-q\right\|^{2} \leq & \zeta_{n}^{1}\left\|x_{n}-q\right\|^{2}+\sum_{k=2}^{K} \zeta_{n}^{k} \prod_{t=1}^{k-1}\left(1-\zeta_{n}^{t}\right)\left\|u_{n}^{k-1}-q\right\|^{2}+\prod_{t=1}^{K}\left(1-\zeta_{n}^{t}\right)\left\|u_{n}^{K}-q\right\|^{2} \\
& -\zeta_{n}^{1}\left[\sum_{k=2}^{K} \zeta_{n}^{k} \prod_{t=1}^{k-1}\left(1-\zeta_{n}^{t}\right)\left\|x_{n}-u_{n}^{k-1}\right\|^{2}+\prod_{t=1}^{K}\left(1-\zeta_{n}^{t}\right)\left\|x_{n}-u_{n}^{K}\right\|^{2}\right] \\
\leq & \zeta_{n}^{1}\left\|x_{n}-q\right\|^{2}+\sum_{k=2}^{K} \zeta_{n}^{k} \prod_{t=1}^{k-1}\left(1-\zeta_{n}^{t}\right) \mathcal{H}^{2}\left(S^{k-1} x_{n}, S^{k-1} q\right) \\
& +\prod_{t=1}^{K}\left(1-\zeta_{n}^{t}\right) \mathcal{H}^{2}\left(S^{K} x_{n}, S^{K} q\right) \\
& \quad-\zeta_{n}^{1}\left[\sum_{k=2}^{K} \zeta_{n}^{k} \prod_{t=1}^{k-1}\left(1-\zeta_{n}^{t}\right)\left\|x_{n}-u_{n}^{k-1}\right\|^{2}+\prod_{t=1}^{K}\left(1-\zeta_{n}^{t}\right)\left\|x_{n}-u_{n}^{K}\right\|^{2}\right] \\
\leq & \zeta_{n}^{1}\left\|x_{n}-q\right\|^{2}+\sum_{k=2}^{K} \zeta_{n}^{k} \prod_{t=1}^{k-1}\left(1-\zeta_{n}^{t}\right)\left[\left\|x_{n}-q\right\|^{2}+\theta^{k-1}\left\|x_{n}-u_{n}^{k-1}\right\|^{2}\right]
\end{aligned}
$$




$$
\begin{aligned}
& +\prod_{t=1}^{K}\left(1-\zeta_{n}^{t}\right)\left[\left\|x_{n}-q\right\|^{2}+\theta^{K}\left\|x_{n}-u_{n}^{K}\right\|^{2}\right] \\
& -\zeta_{n}^{1}\left[\sum_{k=2}^{K} \zeta_{n}^{k} \prod_{t=1}^{k-1}\left(1-\zeta_{n}^{t}\right)\left\|x_{n}-u_{n}^{k-1}\right\|^{2}+\prod_{t=1}^{K}\left(1-\zeta_{n}^{t}\right)\left\|x_{n}-u_{n}^{K}\right\|^{2}\right] \\
= & \left\|x_{n}-q\right\|^{2}-\sum_{j=2}^{K} \zeta_{n}^{k} \prod_{t=1}^{k-1}\left(1-\zeta_{n}^{t}\right)\left(\zeta_{n}^{1}-\theta^{k-1}\right)\left\|x_{n}-u_{n}^{k-1}\right\|^{2} \\
& -\prod_{t=1}^{K}\left(1-\zeta_{n}^{t}\right)\left(\zeta_{n}^{1}-\theta^{K}\right)\left\|x_{n}-u_{n}^{K}\right\|^{2} .
\end{aligned}
$$

Substituting (3.6) into (3.5), we obtain

$$
\begin{aligned}
\left\|m_{n}-q\right\|^{2} \leq & v\left\|x_{n}-q\right\|^{2}-v\left(\zeta_{n}^{1}-\theta^{k-1}\right) \sum_{j=2}^{K} \zeta_{n}^{k} \prod_{t=1}^{k-1}\left(1-\zeta_{n}^{t}\right)\left\|x_{n}-u_{n}^{k-1}\right\|^{2} \\
& -v\left(\zeta_{n}^{1}-\theta^{K}\right) \prod_{t=1}^{K}\left(1-\zeta_{n}^{t}\right)\left\|x_{n}-u_{n}^{K}\right\|^{2}+(1-v)\left\|x_{n}-q\right\|^{2} \\
& -(1-v) \alpha_{n}^{1}\left[\sum_{j=2}^{J} \alpha_{n}^{j} \prod_{t=1}^{j-1}\left(1-\alpha_{n}^{t}\right)\left\|w_{n}-y_{n}^{j-1}\right\|^{2}+\prod_{t=1}^{J}\left(1-\alpha_{n}^{t}\right)\left\|w_{n}-y_{n}^{J}\right\|^{2}\right] \\
\leq & \left\|x_{n}-q\right\|^{2} \\
& -(1-v) \alpha_{n}^{1}\left[\sum_{j=2}^{J} \alpha_{n}^{j} \prod_{t=1}^{j-1}\left(1-\alpha_{n}^{t}\right)\left\|w_{n}-y_{n}^{j-1}\right\|^{2}+\prod_{t=1}^{J}\left(1-\alpha_{n}^{t}\right)\left\|w_{n}-y_{n}^{J}\right\|^{2}\right] \\
& -v\left(\zeta_{n}^{1}-\theta^{k-1}\right) \sum_{j=2}^{K} \zeta_{n}^{k} \prod_{t=1}^{k-1}\left(1-\zeta_{n}^{t}\right)\left\|x_{n}-u_{n}^{k-1}\right\|^{2} \\
& -v\left(\zeta_{n}^{1}-\theta^{K}\right) \prod_{t=1}^{K}\left(1-\zeta_{n}^{t}\right)\left\|x_{n}-u_{n}^{K}\right\|^{2} \\
\leq & \left\|x_{n}-q\right\|^{2} .
\end{aligned}
$$

From (3.1) and (3.7), we obtain

$$
\begin{aligned}
\left\|x_{n+1}-q\right\| & =\left\|\beta_{n}^{1}\left(f_{n}\left(x_{n}\right)-q\right)+\left(1-\beta_{n}^{1}\right)\left[\beta_{n}^{2} x_{n}+\left(1-\beta_{n}^{2}\right) m_{n}-q\right]\right\| \\
& \leq \beta_{n}^{1}\left\|f_{n}\left(x_{n}\right)-q\right\|+\left(1-\beta_{n}^{1}\right) \beta_{n}^{2}\left\|x_{n}-q\right\|+\left(1-\beta_{n}^{1}\right)\left(1-\beta_{n}^{2}\right)\left\|m_{n}-q\right\| \\
& \leq \beta_{n}^{1}\left(\left\|f_{n}\left(x_{n}\right)-f_{n}(q)\right\|+\left\|f_{n}(q)-q\right\|\right)+\left(1-\beta_{n}^{1}\right)\left\|x_{n}-q\right\| .
\end{aligned}
$$

By the uniform convergence of $\left\{f_{n}(x)\right\}$ on any bounded subset $D$ of $H$, and since $\{q\}$ is bounded, there exists $M>0$ such that $\left\|f_{n}(q)-q\right\| \leq M, \forall n \geq 1$. Hence, we have

$$
\begin{aligned}
\left\|x_{n+1}-q\right\| & \leq \beta_{n}^{1} \rho_{n}\left\|x_{n}-q\right\|+\beta_{n}^{1}\left\|f_{n}(q)-q\right\|+\left(1-\beta_{n}^{1}\right)\left\|x_{n}-q\right\| \\
& \leq \beta_{n}^{1} \bar{\rho}\left\|x_{n}-q\right\|+\beta_{n}^{1}\left\|f_{n}(q)-q\right\|+\left(1-\beta_{n}^{1}\right)\left\|x_{n}-q\right\| \\
& =\left(\beta_{n}^{1} \bar{\rho}+\left(1-\beta_{n}^{1}\right)\right)\left\|x_{n}-q\right\|+\beta_{n}^{1}\left\|f_{n}(q)-q\right\|
\end{aligned}
$$




$$
\begin{aligned}
= & \left(1-\beta_{n}^{1}(1-\bar{\rho})\right)\left\|x_{n}-q\right\|+\beta_{n}^{1}\left\|f_{n}(q)-q\right\| \\
= & \left(1-\beta_{n}^{1}(1-\bar{\rho})\right)\left\|x_{n}-q\right\|+\beta_{n}^{1}(1-\bar{\rho}) \frac{\left\|f_{n}(q)-q\right\|}{1-\bar{\rho}} \\
\leq & \max \left\{\left\|x_{n}-q\right\|, \frac{M}{1-\bar{\rho}}\right\} \\
& \vdots \\
\leq & \max \left\{\left\|x_{1}-q\right\|, \frac{M}{1-\bar{\rho}}\right\} .
\end{aligned}
$$

Therefore, $\left\{x_{n}\right\}$ is bounded.

Theorem 3.2 Let $H$ be a real Hilbert space and $E$ be a real uniformly convex and uniformly smooth Banach space. Let $j=1,2, \ldots, J, i=1,2, \ldots, I, k=1,2, \ldots, K$, for some $J, I, K \in \mathbb{N}$. For each $i, j, k$, let $A^{i}: H \rightarrow E$ be a bounded linear operator and $\left(A^{i}\right)^{*}: E^{*} \rightarrow H$ be the adjoint of $A^{i}$, let $M^{j}: H \rightarrow 2^{H}$ be a multivalued maximal monotone mapping, $N^{j}: H \rightarrow H$ be an $\alpha^{j}$-inverse strongly monotone mapping, $S^{k}: H \rightarrow P(H), k=1,2, \ldots, K$ be a finite family of multivalued type-one demicontractive mappings with coefficient $\theta^{k} \in[0,1)$ such that $(I-$ $\left.S^{k}\right)$ is demiclosed at zero, and $Q^{i}: E \rightarrow 2^{E^{*}}$ be a multivalued maximal monotone mapping such that $\Upsilon: \bigcap_{k=1}^{K} F\left(S^{k}\right) \cap \bigcap_{j=1}^{I}\left(M^{j}+N^{j}\right)^{-1}(0) \cap \bigcap_{i=1}^{I}\left(A^{i}\right)^{-1}\left(\left(Q^{i}\right)^{-1}(0)\right) \neq \emptyset$. Let the stepsize $\gamma_{n}^{i}$

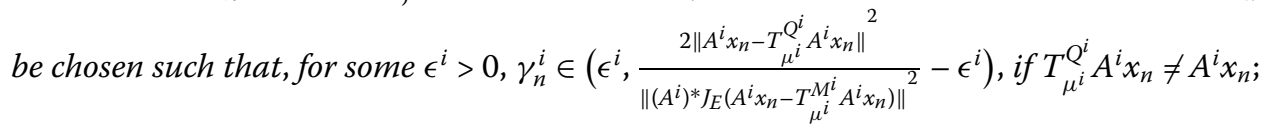
otherwise $\gamma_{n}^{i}=\gamma^{i}$ ( $\gamma^{i}$ being any nonnegative real number). Let $f_{n}: H \rightarrow H$ be a sequence of $\rho_{n}$-contractive mappings with $0<\underline{\rho} \leq \rho_{n} \leq \bar{\rho}<1$ such that $\left\{f_{n}(x)\right\}$ is uniformly convergent for any $x \in D$, where $D$ is any bounded subset of $H$. For arbitrary $x_{1} \in H$, define the sequence $\left\{x_{n}\right\}$ iterative by (3.1), where $\mu>0, v \in(0,1), 0<\lambda^{j} \leq \lambda_{n}^{j}<2 \alpha^{j},\left\{\beta_{n}^{1}\right\}_{n=1}^{\infty},\left\{\beta_{n}^{2}\right\}_{n=1}^{\infty},\left\{\eta_{n}^{i}\right\}_{n=1}^{\infty}$ and $\left\{\zeta_{n}^{k}\right\}_{n=1}^{\infty}$ are real sequences in $(0,1)$ satisfying the following conditions:

(i) $\lim _{n \rightarrow \infty} \beta_{n}^{1}=0$ and $\sum_{n=1}^{\infty} \beta_{n}^{1}=\infty$;

(ii) $0<\epsilon_{1} \leq \beta_{n}^{2}$;

(iii) $\zeta_{n}^{1} \geq \max \left\{\theta^{k}, k=1,2, \ldots, K\right\}, \liminf _{n \rightarrow \infty} \zeta_{n}^{k} \prod_{t=1}^{k-1}\left(1-\zeta_{n}^{t}\right)\left(\zeta_{n}^{1}-\theta^{k-1}\right)>0$,

$$
k=2,3, \ldots,(K-1) \text {; }
$$

(iv) $\liminf _{n \rightarrow \infty} \prod_{t=1}^{K}\left(1-\zeta_{n}^{t}\right)\left(\zeta_{n}^{1}-\theta^{K}\right)>0$;

(v) $\liminf _{n \rightarrow \infty} \eta_{n}^{i} \prod_{t=1}^{i-1}\left(1-\eta_{n}^{t}\right)>0, i=2,3, \ldots,(I-1)$ and $\liminf _{n \rightarrow \infty} \prod_{t=1}^{I}\left(1-\eta_{n}^{t}\right)>0$;

(vi) $\liminf _{n \rightarrow \infty} \alpha_{n}^{j} \prod_{t=1}^{j-1}\left(1-\alpha_{n}^{t}\right)>0, j=2,3, \ldots,(J-1)$ and $\liminf _{n \rightarrow \infty} \prod_{t=1}^{J}\left(1-\alpha_{n}^{t}\right)>0$.

Then the sequence $\left\{x_{n}\right\}$ converges strongly to an element of $\Upsilon$.

Proof Let $q \in \Upsilon$, then from (3.1), (3.7) and Lemma 2.3, we obtain

$$
\begin{aligned}
\left\|x_{n+1}-q\right\|^{2}= & \left\|\beta_{n}^{1}\left(f_{n}\left(x_{n}\right)-f_{n}(q)\right)+\beta_{n}^{1}\left(f_{n}(q)-q\right)+\left(1-\beta_{n}^{1}\right)\left[\beta_{n}^{2} x_{n}+\left(1-\beta_{n}^{2}\right) m_{n}-q\right]\right\|^{2} \\
\leq & \left\|\left(1-\beta_{n}^{1}\right)\left[\beta_{n}^{2} x_{n}+\left(1-\beta_{n}^{2}\right) m_{n}-q\right]+\beta_{n}^{1}\left(f_{n}\left(x_{n}\right)-f_{n}(q)\right)\right\|^{2} \\
& +2 \beta_{n}^{1}\left\langle f_{n}(q)-q, x_{n+1}-q\right\rangle \\
\leq & \left(1-\beta_{n}^{1}\right)^{2}\left\|\beta_{n}^{2} x_{n}+\left(1-\beta_{n}^{2}\right) m_{n}-q\right\|^{2}+\left(\beta_{n}^{1}\right)^{2}\left\|\left(f_{n}\left(x_{n}\right)-f_{n}(q)\right)\right\|^{2} \\
& +2 \beta_{n}^{1}\left(1-\beta_{n}^{1}\right)\left\langle\beta_{n}^{2} x_{n}+\left(1-\beta_{n}^{2}\right) m_{n}-q, f_{n}\left(x_{n}\right)-f_{n}(q)\right\rangle \\
& +2 \beta_{n}^{1}\left\langle f_{n}(q)-q, x_{n+1}-q\right\rangle
\end{aligned}
$$




$$
\begin{aligned}
= & \left(1-\beta_{n}^{1}\right)^{2} \beta_{n}^{2}\left\|x_{n}-q\right\|^{2}+\left(1-\beta_{n}^{1}\right)^{2}\left(1-\beta_{n}^{2}\right)\left\|m_{n}-q\right\|^{2} \\
& -\left(1-\beta_{n}^{1}\right)^{2} \beta_{n}^{2}\left(1-\beta_{n}^{2}\right)\left\|x_{n}-m_{n}\right\|^{2}+\left(\beta_{n}^{1}\right)^{2}\left\|\left(f_{n}\left(x_{n}\right)-f_{n}(q)\right)\right\|^{2} \\
& +2 \beta_{n}^{1}\left(1-\beta_{n}^{1}\right)\left\langle\beta_{n}^{2} x_{n}+\left(1-\beta_{n}^{2}\right) m_{n}-q, f_{n}\left(x_{n}\right)-f_{n}(q)\right\rangle \\
& +2 \beta_{n}^{1}\left(f_{n}(q)-q, x_{n+1}-q\right\rangle \\
\leq & \left(1-\beta_{n}^{1}\right)^{2}\left\|x_{n}-q\right\|^{2}-\left(1-\beta_{n}^{1}\right)^{2} \beta_{n}^{2}\left(1-\beta_{n}^{2}\right)\left\|x_{n}-m_{n}\right\|^{2} \\
& +\left(\beta_{n}^{1}\right)^{2}\left\|f_{n}\left(x_{n}\right)-f_{n}(q)\right\|^{2} \\
& +2 \beta_{n}^{1}\left(1-\beta_{n}^{1}\right)\left\langle\beta_{n}^{2} x_{n}+\left(1-\beta_{n}^{2}\right) m_{n}-q, f_{n}\left(x_{n}\right)-f_{n}(q)\right\rangle \\
& +2 \beta_{n}^{1}\left(f_{n}(q)-q, x_{n+1}-q\right\rangle \\
\leq & \left(1-\beta_{n}^{1}\right)^{2}\left\|x_{n}-q\right\|^{2}-\left(1-\beta_{n}^{1}\right)^{2} \beta_{n}^{2}\left(1-\beta_{n}^{2}\right)\left\|x_{n}-m_{n}\right\|^{2} \\
& +\left(\beta_{n}^{1}\right)^{2} \rho_{n}^{2}\left\|x_{n}-q\right\|^{2} \\
& +2 \beta_{n}^{1}\left(1-\beta_{n}^{1}\right)\left\|\beta_{n}^{2} x_{n}+\left(1-\beta_{n}^{2}\right) m_{n}-q\right\|\left\|f_{n}\left(x_{n}\right)-f_{n}(q)\right\| \\
& +2 \beta_{n}^{1}\left(f_{n}(q)-q, x_{n+1}-q\right\rangle \\
\leq & \left(1-\beta_{n}^{1}\right)^{2}\left\|x_{n}-q\right\|^{2}-\left(1-\beta_{n}^{1}\right)^{2} \beta_{n}^{2}\left(1-\beta_{n}^{2}\right)\left\|x_{n}-m_{n}\right\|^{2} \\
& +\left(\beta_{n}^{1}\right)^{2} \bar{\rho}^{2}\left\|x_{n}-q\right\|^{2}+2 \bar{\rho} \beta_{n}^{1}\left(1-\beta_{n}^{1}\right)\left\|x_{n}-q\right\|^{2} \\
& +2 \beta_{n}^{1}\left(f_{n}(q)-q, x_{n+1}-q\right\rangle .
\end{aligned}
$$

We now divide the rest of the proof into two cases.

Case 1. Suppose that there exists $n_{0} \in \mathbb{N}$ such that $\left\{\left\|x_{n}-q\right\|\right\}_{n=n_{0}}^{\infty}$ is nonincreasing. Then, by Lemma 3.1, $\left\{\left\|x_{n}-q\right\|\right\}$ converges. Thus,

$$
\left\|x_{n}-q\right\|-\left\|x_{n+1}-q\right\| \rightarrow 0 \quad \text { as } n \rightarrow \infty
$$

By (3.1) and (3.6), we obtain

$$
\begin{aligned}
\left\|x_{n+1}-q\right\|^{2} \leq & \beta_{n}^{1}\left\|f_{n}\left(x_{n}\right)-q\right\|^{2}+\left(1-\beta_{n}^{1}\right) \beta_{n}^{2}\left\|x_{n}-q\right\|^{2}+\left(1-\beta_{n}^{1}\right)\left(1-\beta_{n}^{2}\right)\left\|m_{n}-q\right\|^{2} \\
\leq & \beta_{n}^{1}\left\|f_{n}\left(x_{n}\right)-q\right\|^{2}+\left(1-\beta_{n}^{1}\right) \beta_{n}^{2}\left\|x_{n}-q\right\|^{2} \\
& +\left(1-\beta_{n}^{1}\right)\left(1-\beta_{n}^{2}\right)\left(v\left\|u_{n}-q\right\|^{2}+(1-v)\left\|y_{n}-q\right\|^{2}\right) \\
\leq & \beta_{n}^{1}\left\|f_{n}\left(x_{n}\right)-q\right\|^{2}+\left(1-\beta_{n}^{1}\right) \beta_{n}^{2}\left\|x_{n}-q\right\|^{2} \\
& +\left(1-\beta_{n}^{1}\right)\left(1-\beta_{n}^{2}\right)\left(v\left\|x_{n}-q\right\|^{2}+(1-v)\left\|w_{n}-q\right\|^{2}\right) \\
= & \beta_{n}^{1}\left\|f_{n}\left(x_{n}\right)-q\right\|^{2}+\left(1-\beta_{n}^{1}\right)\left(\beta_{n}^{2}+v\left(1-\beta_{n}^{2}\right)\right)\left\|x_{n}-q\right\|^{2} \\
& +\left(1-\beta_{n}^{1}\right)\left(1-\beta_{n}^{2}\right)(1-v)\left\|w_{n}-q\right\|^{2} .
\end{aligned}
$$

By (3.3) and (3.10), we obtain

$$
\begin{aligned}
\eta_{n}^{1} & {\left[\sum_{i=2}^{I} \eta_{n}^{i} \prod_{t=1}^{i-1}\left(1-\eta_{n}^{t}\right)\left\|x_{n}-w_{n}^{i-1}\right\|^{2}+\prod_{t=1}^{I}\left(1-\eta_{n}^{t}\right)\left\|x_{n}-w_{n}^{I}\right\|^{2}\right] } \\
& \leq\left\|x_{n}-q\right\|^{2}-\left\|w_{n}-q\right\|^{2}
\end{aligned}
$$




$$
\begin{aligned}
\leq & \left\|x_{n}-q\right\|^{2}+\frac{\left(\beta_{n}^{2}+v\left(1-\beta_{n}^{2}\right)\right)}{\left(1-\beta_{n}^{2}\right)(1-v)}\left\|x_{n}-q\right\|^{2} \\
& +\frac{1}{\left(1-\beta_{n}^{1}\right)\left(1-\beta_{n}^{2}\right)(1-v)}\left(-\left\|x_{n+1}-q\right\|^{2}+\beta_{n}^{1}\left\|f_{n}\left(x_{n}\right)-q\right\|^{2}\right) \\
= & \frac{\left(1-\beta_{n}^{1}\right)}{\left(1-\beta_{n}^{1}\right)\left(1-\beta_{n}^{2}\right)(1-v)}\left\|x_{n}-q\right\|^{2} \\
& +\frac{1}{\left(1-\beta_{n}^{1}\right)\left(1-\beta_{n}^{2}\right)(1-v)}\left(-\left\|x_{n+1}-q\right\|^{2}+\beta_{n}^{1}\left\|f_{n}\left(x_{n}\right)-q\right\|^{2}\right) \\
= & \frac{1}{\left(1-\beta_{n}^{1}\right)\left(1-\beta_{n}^{2}\right)(1-v)}\left[\left\|x_{n}-q\right\|^{2}-\left\|x_{n+1}-q\right\|^{2}\right] \\
& +\frac{\beta_{n}^{1}}{\left(1-\beta_{n}^{1}\right)\left(1-\beta_{n}^{2}\right)(1-v)}\left[\left\|f_{n}\left(x_{n}\right)-q\right\|^{2}-\left\|x_{n}-q\right\|^{2}\right] .
\end{aligned}
$$

By the conditions on the control sequences, we obtain

$$
\lim _{n \rightarrow \infty}\left\|x_{n}-w_{n}^{i}\right\|=0, \quad i=1,2, \ldots, I
$$

By the condition on $\left\{\gamma_{n}^{i}\right\}$, we obtain

$$
\lim _{n \rightarrow \infty}\left\|\left(A^{i}\right)^{*} J_{E}\left(A^{i} x_{n}-T_{\mu^{i}}^{Q^{i}} A^{i} x_{n}\right)\right\|=0, \quad i=1,2, \ldots, I .
$$

Also, from (3.2), (3.12) and (3.13), we obtain

$$
\begin{aligned}
2 \gamma_{n}^{i}\left\|A^{i} x_{n}-T_{\mu^{i}}^{Q^{i}} A^{i} x_{n}\right\|^{2} \leq & \left\|x_{n}-q\right\|^{2}-\left\|w_{n}^{i}-q\right\|^{2}+\left(\gamma_{n}^{i}\right)^{2}\left\|\left(A^{i}\right)^{*} J_{E}\left(A^{i} x_{n}-T_{\mu^{i}}^{Q^{i}} A^{i} x_{n}\right)\right\|^{2} \\
\leq & \left\|x_{n}-w_{n}^{i}\right\|^{2}-2\left\|x_{n}-w_{n}^{i}\right\|\left\|w_{n}^{i}-q\right\|^{2} \\
& \quad+\left(\gamma_{n}^{i}\right)^{2}\left\|\left(A^{i}\right)^{*} J_{E}\left(A^{i} x_{n}-T_{\mu^{i}}^{Q^{i}} A^{i} x_{n}\right)\right\|^{2} \\
\rightarrow & 0, \quad \text { as } n \rightarrow \infty .
\end{aligned}
$$

Hence,

$$
\lim _{n \rightarrow \infty}\left\|A^{i} x_{n}-T_{\mu^{i}}^{Q^{i}} A^{i} x_{n}\right\|=0, \quad i=1,2, \ldots, I
$$

By (3.8), we have

$$
\left(1-\beta_{n}^{1}\right)^{2} \beta_{n}^{2}\left(1-\beta_{n}^{2}\right)\left\|x_{n}-m_{n}\right\|^{2} \leq\left\|x_{n}-q\right\|^{2}-\left\|x_{n+1}-q\right\|^{2}+\beta_{n}^{1} M_{1},
$$

for some $M_{1}>0$ and this implies that

$$
\lim _{n \rightarrow \infty}\left\|x_{n}-m_{n}\right\|=0
$$

Hence, by (3.1) and condition (i), we obtain

$$
\lim _{n \rightarrow \infty}\left\|x_{n+1}-x_{n}\right\|=0
$$


From (3.7) and (3.16), we obtain

$$
\begin{aligned}
& (1-v) \alpha_{n}^{1}\left[\sum_{j=2}^{J} \alpha_{n}^{j} \prod_{t=1}^{j-1}\left(1-\alpha_{n}^{t}\right)\left\|w_{n}-y_{n}^{j-1}\right\|^{2}+\prod_{t=1}^{J}\left(1-\alpha_{n}^{t}\right)\left\|w_{n}-y_{n}^{J}\right\|^{2}\right] \\
& \leq\left\|x_{n}-q\right\|^{2}-\left\|m_{n}-q\right\|^{2} \rightarrow 0, \quad \text { as } n \rightarrow \infty,
\end{aligned}
$$

which implies from condition (vi) that

$$
\lim _{n \rightarrow \infty}\left\|w_{n}-y_{n}^{j}\right\|=0, \quad j=1,2, \ldots, J
$$

Since $0<\lambda^{j} \leq \lambda_{n}^{j}$, by Lemma 2.6, we obtain

$$
\left\|w_{n}-J_{\lambda^{j}}^{M^{j}}\left(I-\lambda^{j} N^{j}\right) w_{n}\right\| \leq 2\left\|w_{n}-y_{n}^{j}\right\| \rightarrow 0, \quad \text { as } n \rightarrow \infty, j=1,2, \ldots, J
$$

Similarly, we obtain from (3.7) and (3.16)

$$
\begin{aligned}
& v\left(\zeta_{n}^{1}-\theta^{k-1}\right) \sum_{j=2}^{K} \zeta_{n}^{k} \prod_{t=1}^{k-1}\left(1-\zeta_{n}^{t}\right)\left\|x_{n}-u_{n}^{k-1}\right\|^{2}+v\left(\zeta_{n}^{1}-\theta^{K}\right) \prod_{t=1}^{K}\left(1-\zeta_{n}^{t}\right)\left\|x_{n}-u_{n}^{K}\right\|^{2} \\
& \quad \leq\left\|x_{n}-q\right\|^{2}-\left\|m_{n}-q\right\|^{2} \\
& \quad \rightarrow 0, \quad \text { as } n \rightarrow \infty
\end{aligned}
$$

which implies that

$$
\lim _{n \rightarrow \infty}\left\|x_{n}-u_{n}^{k}\right\|=0, \quad k=1,2, \ldots, K
$$

Therefore,

$$
d\left(x_{n}, S^{k} x_{n}\right)=\left\|x_{n}-u_{n}^{k}\right\| \rightarrow 0, \quad n \rightarrow \infty, k=1,2, \ldots, K
$$

Since $\left\{x_{n}\right\}$ is bounded (by Lemma 3.1), there exists a subsequence $\left\{x_{n_{j}}\right\}$ of $\left\{x_{n}\right\}$ such that $x_{n_{j}} \rightarrow x^{*} \in H$, and by the demiclosedness of $\left(I-S^{k}\right)$ (for each $k$ ) at zero and (3.21), we have $x^{*} \in \bigcap_{k=1}^{K} F\left(S^{k}\right)$. Moreover,

$$
\begin{aligned}
\left\|w_{n}-x_{n}\right\|^{2} \leq & \eta_{n}^{1}\left\|x_{n}-x_{n}\right\|^{2}+\sum_{i=2}^{I} \eta_{n}^{i} \prod_{t=1}^{i-1}\left(1-\eta_{n}^{t}\right)\left\|w_{n}^{i-1}-x_{n}\right\|^{2}+\prod_{t=1}^{I}\left(1-\eta_{n}^{t}\right)\left\|w_{n}^{I}-x_{n}\right\|^{2} \\
& -\eta_{n}^{1}\left[\sum_{i=2}^{I} \eta_{n}^{i} \prod_{t=1}^{i-1}\left(1-\eta_{n}^{t}\right)\left\|x_{n}-w_{n}^{i-1}\right\|^{2}+\prod_{t=1}^{I}\left(1-\eta_{n}^{t}\right)\left\|x_{n}-w_{n}^{I}\right\|^{2}\right] \\
\leq & \sum_{i=2}^{I} \eta_{n}^{i} \prod_{t=1}^{i-1}\left(1-\eta_{n}^{t}\right)\left\|w_{n}^{i-1}-x_{n}\right\|^{2}+\prod_{t=1}^{I}\left(1-\eta_{n}^{t}\right)\left\|w_{n}^{I}-x_{n}\right\|^{2}
\end{aligned}
$$

which implies from (3.12) that

$$
\lim _{n \rightarrow \infty}\left\|w_{n}-x_{n}\right\|=0
$$


Furthermore, $A^{i}$ is a bounded linear operator for each $i=1,2, \ldots, I$, so $\left\{A^{i} x_{n_{j}}\right\}$ converges weakly to $A^{i} x^{*} \in E$. Also, $T_{\mu^{i}}^{Q}$ is nonexpansive, thus, by (3.15) and the demiclosedness of $\left(I-T_{\mu^{i}}^{Q_{i}}\right)$ at 0 , we obtain

$$
A x^{*} \in \bigcap_{i=1}^{I} F\left(T_{\mu^{i}}^{Q_{i}}\right) .
$$

Similarly, we obtain from (3.19) and (3.22)

$$
x^{*} \in \bigcap_{j=1}^{J} F\left(J_{\lambda^{j}}^{M_{j}}\left(I-\lambda^{j} N_{j}\right)\right) .
$$

Therefore, we conclude that $x^{*} \in \Upsilon$.

Meanwhile, since $\left\{f_{n}(x)\right\}$ is uniformly convergent on any bounded subset of $H$ and $\left\{x^{*}\right\}$ is bounded, we can set $f\left(x^{*}\right)=\lim _{n \rightarrow \infty} f_{n}\left(x^{*}\right)$. Thus, we obtain

$$
\begin{aligned}
\limsup _{n \rightarrow \infty}\left\langle f_{n}\left(x^{*}\right)-x^{*}, x_{n}-x^{*}\right\rangle & =\limsup _{j \rightarrow \infty}\left\langle f_{n_{j}}\left(x^{*}\right)-x^{*}, x_{n_{j}}-x^{*}\right\rangle \\
& =0 .
\end{aligned}
$$

By replacing q with $x^{*}$ in (3.8), we get

$$
\begin{aligned}
\left\|x_{n+1}-x^{*}\right\|^{2} \leq & \left(1-2 \beta_{n}^{1}\left(1-\bar{\rho}\left(1-\beta_{n}^{1}\right)\right)\right)\left\|x_{n}-x^{*}\right\|^{2}-\left(1-\beta_{n}^{1}\right)^{2} \beta_{n}^{2}\left(1-\beta_{n}^{2}\right)\left\|x_{n}-m_{n}\right\|^{2} \\
& +\left(\beta_{n}^{1}\right)^{2}\left(1+\bar{\rho}^{2}\right)\left\|x_{n}-x^{*}\right\|^{2}+2 \beta_{n}^{1}\left\{f_{n}\left(x^{*}\right)-x^{*}, x_{n+1}-x^{*}\right\rangle \\
\leq & \left(1-2 \beta_{n}^{1}(1-\bar{\rho})\right)\left\|x_{n}-x^{*}\right\|^{2}+2 \beta_{n}^{1}(1-\bar{\rho}) \\
& \times\left[\frac{\beta_{n}^{1}\left(1+\bar{\rho}^{2}\right)}{2(1-\bar{\rho})}\left\|x_{n}-x^{*}\right\|^{2}+\frac{1}{1-\bar{\rho}}\left(\left\langle f_{n}\left(x^{*}\right)-x^{*}, x_{n+1}-x^{*}\right)\right)\right] .
\end{aligned}
$$

Using Lemma 2.4, we obtain $x_{n} \rightarrow x^{*} \in \Upsilon$ as $n \rightarrow \infty$.

Case 2 . Assume that $\left\{\left\|x_{n}-q\right\|\right\}$ is not a monotonically decreasing sequence. Set $\Gamma_{n}=$ $\left\|x_{n}-q\right\|^{2}$ and let $\tau: \mathbb{N} \rightarrow \mathbb{N}$ be a mapping for all $n \geq n_{0}$ (for some $n_{0}$ large enough) by

$$
\tau(n):=\max \left\{k \in \mathbb{N}: k \leq n, \Gamma_{k} \leq \Gamma_{k+1}\right\} .
$$

Clearly, $\tau$ is non-decreasing sequence such that $\tau(n) \rightarrow \infty$ as $n \rightarrow \infty$ and

$$
0 \leq \Gamma_{\tau(n)} \leq \Gamma_{\tau(n)+1}, \quad \forall n \geq n_{0} .
$$

This implies that $\left\|x_{\tau(n)}-q\right\| \leq\left\|x_{\tau(n)+1}-q\right\|, \forall n \geq n_{0}$. Thus $\lim _{n \rightarrow \infty}\left\|x_{\tau(n)}-q\right\|$ exists. In a similar way to Case 1 , we can show that

$$
\left\|\left(A^{i}\right)^{*} J_{E}\left(A^{i} x_{\tau(n)}-T_{\mu^{i}}^{Q^{i}} A^{i} x_{\tau(n)}\right)\right\| \rightarrow 0, \quad n \rightarrow \infty, i=1,2, \ldots, I .
$$

Similarly,

$$
\left\|x_{\tau(n)}-u_{\tau(n)}^{k}\right\| \rightarrow 0, \quad n \rightarrow \infty, k=1,2, \ldots, K,
$$


so that

$$
d\left(x_{\tau(n)}, S^{k} x_{\tau(n)}\right)=\left\|x_{\tau(n)}-u_{\tau(n)}^{k}\right\| \rightarrow 0, \quad n \rightarrow \infty, k=1,2, \ldots, K .
$$

We can also show that

$$
\begin{aligned}
& \left\|x_{\tau(n)+1}-x_{\tau(n)}\right\| \rightarrow 0, \quad n \rightarrow \infty, \\
& \left\|w_{\tau(n)}-x_{\tau(n)}\right\| \rightarrow 0, \quad n \rightarrow \infty, \\
& \left\|A^{i} x_{\tau(n)}-T_{\mu^{i}}^{Q^{i}} A^{i} x_{\tau(n)}\right\| \rightarrow 0, \quad n \rightarrow \infty, i=1,2, \ldots, I .
\end{aligned}
$$

and

$$
\left\|w_{\tau(n)}-J_{\lambda^{j}}^{M^{j}}\left(I-\lambda^{j} N^{j}\right) w_{\tau(n)}\right\| \rightarrow 0, \quad \text { as } n \rightarrow \infty, j=1,2, \ldots, J
$$

From the fact that $\left\{x_{\tau(n)}\right\}$ is bounded, we see that there exists a subsequence of $\left\{x_{\tau(n)}\right\}$, denoted as $\left\{x_{\tau(n)}\right\}$, that converges weakly to $x^{*} \in H_{1}$. Since $\left\|w_{\tau(n)}-x_{\tau(n)}\right\| \rightarrow 0$, it follows that $w_{\tau(n)} \rightarrow x^{*} \in H_{1}$. As in Case 1 , we can show that $x^{*} \in \Upsilon$ and

$$
\limsup _{n \rightarrow \infty}\left\langle f_{\tau(n)}\left(x^{*}\right)-x^{*}, x_{\tau(n)+1}-x^{*}\right\rangle=0 .
$$

By replacing $q$ with $x^{*}$ in (3.8), we get

$$
\begin{aligned}
\left\|x_{\tau(n)+1}-x^{*}\right\|^{2} \leq & \left(1-2 \beta_{\tau(n)}^{1}\left(1-\bar{\rho}\left(1-\beta_{\tau(n)}^{1}\right)\right)\right)\left\|x_{\tau(n)}-x^{*}\right\|^{2} \\
& -\left(1-\beta_{\tau(n)}^{1}\right)^{2} \beta_{\tau(n)}^{2}\left(1-\beta_{\tau(n)}^{2}\right)\left\|x_{\tau}(n)-m_{\tau}(n)\right\|^{2} \\
& +\left(\beta_{\tau(n)}^{1}\right)^{2}\left(1+\bar{\rho}^{2}\right)\left\|x_{\tau(n)}-x^{*}\right\|^{2}+2 \beta_{\tau(n)}^{1}\left\langle f_{\tau(n)}\left(x^{*}\right)-x^{*}, x_{\tau(n)+1}-x^{*}\right\rangle \\
\leq & \left(1-2 \beta_{\tau(n)}^{1}(1-\bar{\rho})\right)\left\|x_{\tau(n)}-x^{*}\right\|^{2}+\left(\beta_{\tau(n)}^{1}\right)^{2}\left(1+\bar{\rho}^{2}\right)\left\|x_{\tau(n)}-x^{*}\right\|^{2} \\
& +2 \beta_{\tau(n)}^{1}\left(f_{\tau(n)}\left(x^{*}\right)-x^{*}, x_{\tau(n)+1}-x^{*}\right\rangle
\end{aligned}
$$

This implies that (noting that $\Gamma_{\tau(n)} \leq \Gamma_{\tau(n)+1}$ and $\beta_{\tau(n)}^{1}>0$ )

$$
2(1-\bar{\rho})\left\|x_{\tau(n)}-x^{*}\right\|^{2} \leq \beta_{\tau(n)}^{1}\left(1+\bar{\rho}^{2}\right)\left\|x_{\tau(n)}-x^{*}\right\|^{2}+2\left\langle f_{\tau(n)}\left(x^{*}\right)-x^{*}, x_{\tau(n)+1}-x^{*}\right) .
$$

This implies that

$$
\limsup _{n \rightarrow \infty}\left\|x_{\tau(n)}-x^{*}\right\| \leq 0
$$

Thus,

$$
\lim _{n \rightarrow \infty}\left\|x_{\tau(n)}-x^{*}\right\|=0
$$

Therefore,

$$
\left\|x_{\tau(n)+1}-x^{*}\right\| \leq\left\|x_{\tau(n)}-x^{*}\right\|+\left\|x_{\tau(n)+1}-x_{\tau(n)}\right\| \rightarrow 0, \quad n \rightarrow \infty .
$$


Furthermore, for $n \geq n_{0}$, it is easy to see that $\Gamma_{\tau(n)} \leq \Gamma_{\tau(n)+1}$ if $n \neq \tau(n)$ (that is, $\left.\tau(n)<n\right)$, because $\Gamma_{j} \leq \Gamma_{j+1}$ for $\tau(n)+1 \leq j \leq n$. As a consequence, we obtain for all $n \geq n_{0}$,

$$
0 \leq \Gamma_{n} \leq \max \left\{\Gamma_{\tau(n)}, \Gamma_{\tau(n)+}\right\}=\Gamma_{\tau(n)+1} .
$$

Hence, $\lim _{n \rightarrow \infty} \Gamma_{n}=0$, that is, $\left\{x_{n}\right\}$ converges to $x^{*} \in \Upsilon$. This completes the proof.

The following new results follow directly from Theorem 3.2.

Corollary 3.3 Let $H$ be a real Hilbert space and $E$ be a real uniformly convex and uniformly smooth Banach space. Let $i=1,2, \ldots, I, k=1,2, \ldots, K$, for some $I, K \in \mathbb{N}$. For each $i, k$, let $A^{i}: H \rightarrow E$ be a bounded linear operator and $\left(A^{i}\right)^{*}: E^{*} \rightarrow H$ be the adjoint of $A^{i}, M: H \rightarrow 2^{H}$ be a multivalued maximal monotone mapping and $N: H \rightarrow H$ be an $\alpha$-inverse strongly monotone mapping and $S^{k}: H \rightarrow P(H), k=1,2, \ldots, K$ be a finite family of multivalued type-one demicontractive mappings with coefficient $\theta^{k} \in[0,1)$ such that $\left(I-S^{k}\right)$ is demiclosed at zero, and $Q^{i}: E \rightarrow 2^{E^{*}}$ be a multivalued maximal monotone mapping such that $\Upsilon: \bigcap_{k=1}^{K} F\left(S^{k}\right) \cap(M+N)^{-1}(0) \cap \bigcap_{i=1}^{I}\left(A^{i}\right)^{-1}\left(\left(Q^{i}\right)^{-1}(0)\right) \neq \emptyset$. Let the stepsize $\gamma_{n}^{i}$ be chosen such that, for some $\epsilon^{i}>0, \gamma_{n}^{i} \in\left(\epsilon^{i}, \frac{2\left\|A^{i} x_{n}-T_{\mu}^{Q^{i}} A^{i} x_{n}\right\|^{2}}{\left\|\left(A^{i}\right)^{*} E_{E}\left(A^{i} x_{x_{n}}-T_{\mu}^{M^{i}} A^{i} x_{n}\right)\right\|^{2}}-\epsilon^{i}\right)$, if $T_{\mu}^{Q^{i}} A^{i} x_{n} \neq A^{i} x_{n}$; otherwise $\gamma_{n}^{i}=\gamma^{i}$ ( $\gamma^{i}$ being any nonnegative real number). Let $f_{n}: H \rightarrow H$ be a sequence of $\rho_{n}$-contractive mappings with $0<\rho \leq \rho_{n} \leq \bar{\rho}<1$ such that $\left\{f_{n}(x)\right\}$ is uniformly convergent for any $x \in D$, where $D$ is any bounded subset of $H$. For arbitrary $x_{1} \in H$, define the iterative sequence $\left\{x_{n}\right\}$ by

$$
\left\{\begin{array}{l}
w_{n}^{i}=x_{n}-\gamma_{n}\left(A^{i}\right)^{*} J_{E}\left(A^{i} x_{n}-T_{\mu}^{Q^{i}} A^{i} x_{n}\right), \quad i-1,2, \ldots, I, \\
w_{n}=\eta_{n}^{1} x_{n}+\sum_{i=2}^{I} \eta_{n}^{i} \prod_{t=1}^{i-1}\left(1-\eta_{n}^{t}\right) w_{n}^{i-1}+\prod_{i=1}^{I}\left(1-\eta_{n}^{i}\right) w^{I}, \\
y_{n}=J_{\lambda_{n}}^{M}\left(I-\lambda_{n} N\right) w_{n}, \\
u_{n}=\zeta_{n}^{1} x_{n}+\sum_{k=2}^{K} \zeta_{n}^{k} \prod_{t=1}^{k-1}\left(1-\zeta_{n}^{t}\right) u_{n}^{k-1}+\prod_{k=1}^{K}\left(1-\zeta_{n}^{k}\right) u_{n}^{K}, \\
x_{n+1}=\beta_{n}^{1} f_{n}\left(x_{n}\right)+\left(1-\beta_{n}^{1}\right)\left[\beta_{n}^{2} x_{n}+\left(1-\beta_{n}^{2}\right)\left(v u_{n}+(1-v) y_{n}\right)\right],
\end{array}\right.
$$

where $u_{n}^{k} \in P_{S^{k}} x_{n}:=\left\{u_{n}^{k} \in S^{k} x_{n}:\left\|u_{n}^{k}-x_{n}\right\|=d\left(x_{n}, S^{k} x_{n}\right)\right\}, k=1,2, \ldots, K, \mu>0, v \in(0,1)$, $0<\lambda \leq \lambda_{n}<2 \alpha,\left\{\beta_{n}^{1}\right\}_{n=1}^{\infty},\left\{\beta_{n}^{2}\right\}_{n=1}^{\infty},\left\{\eta_{n}^{i}\right\}_{n=1}^{\infty}$ and $\left\{\zeta_{n}^{k}\right\}_{n=1}^{\infty}$ are real sequences in $(0,1)$ satisfying the following conditions:

(i) $\lim _{n \rightarrow \infty} \beta_{n}^{1}=0$ and $\sum_{n=1}^{\infty} \beta_{n}^{1}=\infty$;

(ii) $0<\epsilon_{1} \leq \beta_{n}^{2}$;

(iii) $\zeta_{n}^{1} \geq \max \left\{\theta^{k}, k=1,2, \ldots, K\right\}, \liminf _{n \rightarrow \infty} \zeta_{n}^{k} \prod_{t=1}^{k-1}\left(1-\zeta_{n}^{t}\right)\left(\zeta_{n}^{1}-\theta^{k-1}\right)>0$, $k=2,3, \ldots,(K-1)$;

(iv) $\liminf _{n \rightarrow \infty} \prod_{t=1}^{K}\left(1-\zeta_{n}^{t}\right)\left(\zeta_{n}^{1}-\theta^{K}\right)>0$;

(v) $\liminf _{n \rightarrow \infty} \eta_{n}^{i} \prod_{t=1}^{i-1}\left(1-\eta_{n}^{t}\right)>0, i=2,3, \ldots,(I-1)$ and $\liminf _{n \rightarrow \infty} \prod_{t=1}^{I}\left(1-\eta_{n}^{t}\right)>0$.

Then the sequence $\left\{x_{n}\right\}$ converges strongly to an element of $\Upsilon$.

Corollary 3.4 Let $H$ be a real Hilbert space and $E$ be a real uniformly convex and uniformly smooth Banach space. Let $j=1,2, \ldots, J, k=1,2, \ldots, K$, for some $J, K \in \mathbb{N}$. For each $j$, $k$, let $A: H \rightarrow E$ be a bounded linear operator and $A^{*}: E^{*} \rightarrow H$ be the adjoint of $A$, 
$M^{j}: H \rightarrow 2^{H}$ be a multivalued maximal monotone mapping and $N^{j}: H \rightarrow H$ be an $\alpha$ inverse strongly monotone mapping and $S^{k}: H \rightarrow P(H), k=1,2, \ldots, K$ be a finite family of multivalued type-one demicontractive mappings with coefficient $\theta^{k} \in[0,1)$ such that $\left(I-S^{k}\right)$ is demiclosed at zero, and $Q: E \rightarrow 2^{E^{*}}$ be a multivalued maximal monotone mapping such that $\Upsilon: \bigcap_{k=1}^{K} F\left(S^{k}\right) \cap \bigcap_{j=1}^{J}\left(M^{j}+N^{j}\right)^{-1}(0) \cap A^{-1}\left(Q^{-1}(0)\right) \neq \emptyset$. Let the stepsize $\gamma_{n}$ be chosen such that, for some $\epsilon>0, \gamma_{n} \in\left(\epsilon, \frac{2\left\|A x_{n}-T_{\mu}^{Q} A x_{n}\right\|^{2}}{\left\|(A)^{*} J_{E}\left(A x_{n}-T_{\mu}^{M} A x_{n}\right)\right\|^{2}}-\epsilon\right)$, if $T_{\mu}^{Q} A x_{n} \neq A x_{n}$; otherwise $\gamma_{n}=\gamma$ ( $\gamma$ being any nonnegative real number). Let $f_{n}: H \rightarrow H$ be a sequence of $\rho_{n}$-contractive mappings with $0<\underline{\rho} \leq \rho_{n} \leq \bar{\rho}<1$ such that $\left\{f_{n}(x)\right\}$ is uniformly convergent for any $x \in D$, where $D$ is any bounded subset of $H$. For arbitrary $x_{1} \in H$, define the iterative sequence $\left\{x_{n}\right\}$ by

$$
\left\{\begin{array}{l}
w_{n}=x_{n}-\gamma_{n} A^{*} J_{E}\left(A x_{n}-T_{\mu}^{Q} A x_{n}\right), \\
y_{n}^{j}=J_{\lambda_{n}^{j}}^{M^{j}}\left(I-\lambda_{n}^{j} N^{j}\right) w_{n}, \quad j=1,2, \ldots, J, \\
y_{n}=\alpha_{n}^{1} w_{n}+\sum_{j=2}^{J} \alpha_{n}^{j} \prod_{t=1}^{j-1}\left(1-\alpha_{n}^{t}\right) y_{n}^{j-1}+\prod_{j=1}^{J}\left(1-\alpha_{n}^{j}\right) y^{J}, \\
u_{n}=\zeta_{n}^{1} x_{n}+\sum_{k=2}^{K} \zeta_{n}^{k} \prod_{t=1}^{k-1}\left(1-\zeta_{n}^{t}\right) u_{n}^{k-1}+\prod_{k=1}^{K}\left(1-\zeta_{n}^{k}\right) u_{n}^{K}, \\
x_{n+1}=\beta_{n}^{1} f_{n}\left(x_{n}\right)+\left(1-\beta_{n}^{1}\right)\left[\beta_{n}^{2} x_{n}+\left(1-\beta_{n}^{2}\right)\left(v u_{n}+(1-v) y_{n}\right)\right],
\end{array}\right.
$$

where $u_{n}^{k} \in P_{S^{k}} x_{n}:=\left\{u_{n}^{k} \in S^{k} x_{n}:\left\|u_{n}^{k}-x_{n}\right\|=d\left(x_{n}, S^{k} x_{n}\right)\right\}, k=1,2, \ldots, K, \mu>0, v \in(0,1)$, $0<\lambda^{j} \leq \lambda_{n}^{j}<2 \alpha^{j},\left\{\beta_{n}^{1}\right\}_{n=1}^{\infty},\left\{\beta_{n}^{2}\right\}_{n=1}^{\infty},\left\{\alpha_{n}^{j}\right\}_{n=1}^{\infty}$ and $\left\{\zeta_{n}^{k}\right\}_{n=1}^{\infty}$ are real sequences in $(0,1)$ satisfying the following conditions:

(i) $\lim _{n \rightarrow \infty} \beta_{n}^{1}=0$ and $\sum_{n=1}^{\infty} \beta_{n}^{1}=\infty$;

(ii) $0<\epsilon_{1} \leq \beta_{n}^{2}$;

(iii) $\zeta_{n}^{1} \geq \max \left\{\theta^{k}, k=1,2, \ldots, K\right\}, \liminf _{n \rightarrow \infty} \zeta_{n}^{k} \prod_{t=1}^{k-1}\left(1-\zeta_{n}^{t}\right)\left(\zeta_{n}^{1}-\theta^{k-1}\right)>0$, $k=2,3, \ldots,(K-1)$;

(iv) $\liminf _{n \rightarrow \infty} \prod_{t=1}^{K}\left(1-\zeta_{n}^{t}\right)\left(\zeta_{n}^{1}-\theta^{K}\right)>0$;

(v) $\liminf _{n \rightarrow \infty} \alpha_{n}^{j} \prod_{t=1}^{j-1}\left(1-\alpha_{n}^{t}\right)>0, j=2,3, \ldots,(J-1)$ and $\liminf _{n \rightarrow \infty} \prod_{t=1}^{J}\left(1-\alpha_{n}^{t}\right)>0$.

Then the sequence $\left\{x_{n}\right\}$ converges strongly to an element of $\Upsilon$.

Corollary 3.5 Let $H$ be a real Hilbert space and $E$ be a real uniformly convex and uniformly smooth Banach space. Let $j=1,2, \ldots, J, i=1,2, \ldots, I$, for some $J, I \in \mathbb{N}$.For each $i$, j, let $A^{i}: H \rightarrow E$ be a bounded linear operator and $\left(A^{i}\right)^{*}: E^{*} \rightarrow H$ be the adjoint of $A^{i}, M^{j}: H \rightarrow 2^{H}$ be a multivalued maximal monotone mapping and $N^{j}: H \rightarrow H$ be an $\alpha$-inverse strongly monotone mapping. Suppose $S: H \rightarrow P(H)$ is a multivalued type-one demicontractive mapping with coefficient $\theta \in[0,1)$ such that $(I-S)$ is demiclosed at zero and $Q^{i}: E \rightarrow 2^{E^{*}}$ be a multivalued maximal monotone mapping such that $\Upsilon: F(S) \cap \bigcap_{j=1}^{J}\left(M^{j}+N^{j}\right)^{-1}(0) \cap \bigcap_{i=1}^{I}\left(A^{i}\right)^{-1}\left(\left(Q^{i}\right)^{-1}(0)\right) \neq \emptyset$. Let the stepsize $\gamma_{n}^{i}$ be chosen such that, for some $\epsilon^{i}>0, \gamma_{n}^{i} \in\left(\epsilon^{i}, \frac{2\left\|A^{i} x_{n}-T_{\mu}^{Q^{i}} A^{i} x_{n}\right\|^{2}}{\left\|\left(A^{i}\right)^{*} J_{E}\left(A^{i} x_{n}-T_{\mu^{i}}^{M^{i}} A^{i} x_{n}\right)\right\|^{2}}-\epsilon^{i}\right)$, if $T_{\mu^{i}}^{Q^{i}} A^{i} x_{n} \neq A^{i} x_{n}$; otherwise $\gamma_{n}^{i}=\gamma^{i}$ ( $\gamma^{i}$ being any nonnegative real number). Let $f_{n}: H \rightarrow H$ be a sequence of $\rho_{n^{-}}$ contractive mappings with $0<\underline{\rho} \leq \rho_{n} \leq \bar{\rho}<1$ such that $\left\{f_{n}(x)\right\}$ is uniformly convergent for any $x \in D$, where $D$ is any bounded subset of $H$. For arbitrary $x_{1} \in H$, define the iterative 
sequence $\left\{x_{n}\right\}$ by

$$
\left\{\begin{array}{l}
w_{n}^{i}=x_{n}-\gamma_{n}\left(A^{i}\right)^{*} J_{E}\left(A^{i} x_{n}-T_{\mu}^{Q^{i}} A^{i} x_{n}\right), \quad i=1,2, \ldots, I, \\
w_{n}=\eta_{n}^{1} x_{n}+\sum_{i=2}^{I} \eta_{n}^{i} \prod_{t=1}^{i-1}\left(1-\eta_{n}^{t}\right) w_{n}^{i-1}+\prod_{i=1}^{I}\left(1-\eta_{n}^{i}\right) w^{I}, \\
y_{n}^{j}=J_{\lambda_{n}^{j}}^{M^{j}}\left(I-\lambda_{n}^{j} N^{j}\right) w_{n}, \quad j=1,2, \ldots, J, \\
y_{n}=\alpha_{n}^{1} w_{n}+\sum_{j=2}^{J} \alpha_{n}^{j} \prod_{t=1}^{j-1}\left(1-\alpha_{n}^{t}\right) y_{n}^{j-1}+\prod_{j=1}^{J}\left(1-\alpha_{n}^{j}\right) y^{J}, \\
x_{n+1}=\beta_{n}^{1} f_{n}\left(x_{n}\right)+\left(1-\beta_{n}^{1}\right)\left[\beta_{n}^{2} x_{n}+\left(1-\beta_{n}^{2}\right)\left(v u_{n}+(1-v) y_{n}\right)\right],
\end{array}\right.
$$

where $u_{n} \in P_{S} x_{n}:=\left\{u_{n} \in S x_{n}:\left\|u_{n}-x_{n}\right\|=d\left(x_{n}, S x_{n}\right)\right\}, \mu>0, v \in(\theta, 1), 0<\lambda^{j} \leq \lambda_{n}^{j}<2 \alpha^{j}$, $\left\{\beta_{n}^{1}\right\}_{n=1}^{\infty},\left\{\beta_{n}^{2}\right\}_{n=1}^{\infty},\left\{\alpha_{n}^{j}\right\}_{n=1}^{\infty}$ and $\left\{\eta_{n}^{i}\right\}_{n=1}^{\infty}$ are real sequences in $(0,1)$ satisfying the following conditions:

(i) $\lim _{n \rightarrow \infty} \beta_{n}^{1}=0$ and $\sum_{n=1}^{\infty} \beta_{n}^{1}=\infty$;

(ii) $0<\epsilon_{1} \leq \beta_{n}^{2} \leq 1-\theta$;

(iii) $\liminf _{n \rightarrow \infty} \eta_{n}^{i} \prod_{t=1}^{i-1}\left(1-\eta_{n}^{t}\right)>0, i=2,3, \ldots,(I-1)$ and $\liminf _{n \rightarrow \infty} \prod_{t=1}^{I}\left(1-\eta_{n}^{t}\right)>0$;

(iv) $\liminf _{n \rightarrow \infty} \alpha_{n}^{j} \prod_{t=1}^{j-1}\left(1-\alpha_{n}^{t}\right)>0, j=2,3, \ldots,(J-1)$ and $\liminf _{n \rightarrow \infty} \prod_{t=1}^{J}\left(1-\alpha_{n}^{t}\right)>0$.

Then the sequence $\left\{x_{n}\right\}$ converges strongly to an element of $\Upsilon$.

Corollary 3.6 Let $H$ be a real Hilbert space and $E$ be a real uniformly convex and uniformly smooth Banach space. Let $j=1,2, \ldots, J$, for some $J \in \mathbb{N}$. For each $j$, let $M^{j}: H \rightarrow 2^{H}$ be a multivalued maximal monotone mapping and $N^{j}: H \rightarrow H$ be an $\alpha$-inverse strongly monotone mapping $A: H \rightarrow E$ be a bounded linear operator and $A^{*}: E^{*} \rightarrow H$ be the adjoint of $A$, and $S: H \rightarrow P(H)$ be a multivalued type-one demicontractive mapping with coefficient $\theta \in[0,1)$ such that $(I-S)$ is demiclosed at zero, and $Q: E \rightarrow 2^{E^{*}}$ be a multivalued maximal monotone mapping such that $\Upsilon: F(S) \cap \bigcap_{j=1}^{J}\left(M^{j}+N^{j}\right)^{-1}(0) \cap A^{-1}\left(Q^{-1}(0)\right) \neq \emptyset$. Let the stepsize $\gamma_{n}$ be chosen such that, for some $\epsilon>0, \gamma_{n} \in\left(\epsilon, \frac{2\left\|A x_{n}-T_{\mu}^{Q} A x_{n}\right\|^{2}}{\left\|(A)^{*} J_{E}\left(A x_{n}-T_{\mu}^{M} A x_{n}\right)\right\|^{2}}-\epsilon\right)$, if $T_{\mu}^{Q} A x_{n} \neq A x_{n}$; otherwise $\gamma_{n}=\gamma$ ( $\gamma$ being any nonnegative real number). Let $f_{n}: H \rightarrow H$ be a sequence of $\rho_{n}$-contractive mappings with $0<\underline{\rho} \leq \rho_{n} \leq \bar{\rho}<1$ such that $\left\{f_{n}(x)\right\}$ is uniformly convergent for any $x \in D$, where $D$ is any bounded subset of $H$. For arbitrary $x_{1} \in H$, define the iterative sequence $\left\{x_{n}\right\}$ by

$$
\left\{\begin{array}{l}
w_{n}=x_{n}-\gamma_{n}(A)^{*} J_{E}\left(A x_{n}-T_{\mu}^{Q} A x_{n}\right), \\
y_{n}^{j}=J_{\lambda_{n}^{j}}^{M^{j}}\left(I-\lambda_{n}^{j} N^{j}\right) w_{n}, \quad j=1,2, \ldots, J, \\
y_{n}=\alpha_{n}^{1} w_{n}+\sum_{j=2}^{J} \alpha_{n}^{j} \prod_{t=1}^{j-1}\left(1-\alpha_{n}^{t}\right) y_{n}^{j-1}+\prod_{j=1}^{J}\left(1-\alpha_{n}^{j}\right) y^{J}, \\
x_{n+1}=\beta_{n}^{1} f_{n}\left(x_{n}\right)+\left(1-\beta_{n}^{1}\right)\left[\beta_{n}^{2} x_{n}+\left(1-\beta_{n}^{2}\right)\left(v u_{n}+(1-v) y_{n}\right)\right],
\end{array}\right.
$$

where $u_{n} \in P_{S} x_{n}:=\left\{u_{n} \in S x_{n}:\left\|u_{n}-x_{n}\right\|=d\left(x_{n}, S x_{n}\right)\right\}, \mu>0, v \in(\theta, 1), 0<\lambda^{j} \leq \lambda_{n}^{j}<2 \alpha^{j}$, $\left\{\beta_{n}^{1}\right\}_{n=1}^{\infty},\left\{\beta_{n}^{2}\right\}_{n=1}^{\infty}$, and $\left\{\alpha_{n}^{j}\right\}_{n=1}^{\infty}$ are real sequences in $(0,1)$ satisfying the following conditions:

(i) $\lim _{n \rightarrow \infty} \beta_{n}^{1}=0$ and $\sum_{n=1}^{\infty} \beta_{n}^{1}=\infty$;

(ii) $0<\epsilon_{1} \leq \beta_{n}^{2} \leq 1-\theta$;

(iii) $\liminf _{n \rightarrow \infty} \alpha_{n}^{j} \prod_{t=1}^{j-1}\left(1-\alpha_{n}^{t}\right)>0, j=2,3, \ldots,(J-1)$ and $\liminf _{n \rightarrow \infty} \prod_{t=1}^{J}\left(1-\alpha_{n}^{t}\right)>0$.

Then the sequence $\left\{x_{n}\right\}$ converges strongly to an element of $\Upsilon$.

Corollary 3.7 Let $H$ be a real Hilbert space and $E$ be a real uniformly convex and uniformly smooth Banach space. Let $k=1,2, \ldots, K$, for some $K \in \mathbb{N}$. For each $k$, let $S^{k}$ : 
$H \rightarrow P(H), k=1,2, \ldots, K$ be a finite family of multivalued type-one demicontractive mappings with coefficient $\theta^{k} \in[0,1)$ such that $\left(I-S^{k}\right)$ is demiclosed at zero, $A: H \rightarrow E$ be a bounded linear operator and $A^{*}: E^{*} \rightarrow H$ be the adjoint of $A, M: H \rightarrow 2^{H}$ be a multivalued maximal monotone mapping and $N: H \rightarrow H$ be an $\alpha$-inverse strongly monotone mapping, and $Q: E \rightarrow 2^{E^{*}}$ be a multivalued maximal monotone mapping such that $\Upsilon: \bigcap_{k=1}^{K} F\left(S^{k}\right) \cap(M+N)^{-1}(0) \cap A^{-1}\left(Q^{-1}(0)\right) \neq \emptyset$. Let the stepsize $\gamma_{n}$ be chosen such that, for some $\epsilon>0, \gamma_{n} \in\left(\epsilon, \frac{2\left\|A x_{n}-T_{\mu}^{Q} A x_{n}\right\|^{2}}{\left\|(A)^{*} J_{E}\left(A x_{n}-T_{\mu}^{M} A x_{n}\right)\right\|^{2}}-\epsilon\right)$, if $T_{\mu}^{Q} A x_{n} \neq A x_{n}$; otherwise $\gamma_{n}=\gamma(\gamma$ being any nonnegative real number). Let $f_{n}: H \rightarrow H$ be a sequence of $\rho_{n}$-contractive mappings with $0<\underline{\rho} \leq \rho_{n} \leq \bar{\rho}<1$ such that $\left\{f_{n}(x)\right\}$ is uniformly convergent for any $x \in D$, where $D$ is any bounded subset of $H$. For arbitrary $x_{1} \in H$, define the iterative sequence $\left\{w_{n}\right\},\left\{x_{n}\right\}$ and $\left\{y_{n}\right\}$ by

$$
\left\{\begin{array}{l}
w_{n}=x_{n}-\gamma_{n} A^{*} J_{E}\left(A x_{n}-T_{\mu}^{Q} A x_{n}\right), \\
y_{n}=J_{\lambda_{n}}^{M}\left(I-\lambda_{n} N\right) w_{n}, \\
u_{n}=\zeta_{n}^{1} x_{n}+\sum_{k=2}^{K} \zeta_{n}^{k} \prod_{t=1}^{k-1}\left(1-\zeta_{n}^{t}\right) u_{n}^{k-1}+\prod_{k=1}^{K}\left(1-\zeta_{n}^{k}\right) u_{n}^{K}, \\
x_{n+1}=\beta_{n}^{1} f_{n}\left(x_{n}\right)+\left(1-\beta_{n}^{1}\right)\left[\beta_{n}^{2} x_{n}+\left(1-\beta_{n}^{2}\right)\left(v u_{n}+(1-v) y_{n}\right)\right],
\end{array}\right.
$$

where $u_{n}^{k} \in P_{S^{k}} x_{n}:=\left\{u_{n}^{k} \in S^{k} x_{n}:\left\|u_{n}^{k}-x_{n}\right\|=d\left(x_{n}, S^{k} x_{n}\right)\right\}, k=1,2, \ldots, K, \mu>0, v \in(0,1), 0<$ $\lambda \leq \lambda_{n}<2 \alpha,\left\{\beta_{n}^{1}\right\}_{n=1}^{\infty},\left\{\beta_{n}^{2}\right\}_{n=1}^{\infty}$, and $\left\{\zeta_{n}^{k}\right\}_{n=1}^{\infty}$ are real sequences in $(0,1)$ satisfying the following conditions:

(i) $\lim _{n \rightarrow \infty} \beta_{n}^{1}=0$ and $\sum_{n=1}^{\infty} \beta_{n}^{1}=\infty$;

(ii) $0<\epsilon_{1} \leq \beta_{n}^{2}$;

(iii) $\zeta_{n}^{1} \geq \max \left\{\theta^{k}, k=1,2, \ldots, K\right\}, \liminf _{n \rightarrow \infty} \zeta_{n}^{k} \prod_{t=1}^{k-1}\left(1-\zeta_{n}^{t}\right)\left(\zeta_{n}^{1}-\theta^{k-1}\right)>0$,

$$
k=2,3, \ldots,(K-1) \text {; }
$$

(iv) $\liminf _{n \rightarrow \infty} \prod_{t=1}^{K}\left(1-\zeta_{n}^{t}\right)\left(\zeta_{n}^{1}-\theta^{K}\right)>0$.

Then the sequence $\left\{x_{n}\right\}$ converges strongly to an element of $\Upsilon$.

Corollary 3.8 Let $H$ be a real Hilbert space and $E$ be a real uniformly convex and uniformly smooth Banach space. Let $i=1,2, \ldots, I$, for some $I \in \mathbb{N}$. For each $i, k$, let $A^{i}$ : $H \rightarrow E$ be a bounded linear operator and $\left(A^{i}\right)^{*}: E^{*} \rightarrow H$ be the adjoint of $A^{i}$. Suppose $M: H \rightarrow 2^{H}$ be a multivalued maximal monotone mapping and $N: H \rightarrow H$ be an $\alpha$ inverse strongly monotone mapping and $S: H \rightarrow P(H)$ be a multivalued type-one demicontractive mapping with coefficient $\theta \in[0,1)$ such that $(I-S)$ is demiclosed at zero, and $Q^{i}: E \rightarrow 2^{E^{*}}$ be a multivalued maximal monotone mapping such that $\Upsilon: F(S) \cap(M+$ $N)^{-1}(0) \cap \bigcap_{i=1}^{I}\left(A^{i}\right)^{-1}\left(\left(Q^{i}\right)^{-1}(0)\right) \neq \emptyset$. Let the stepsize $\gamma_{n}^{i}$ be chosen such that, for some $\epsilon^{i}>0$, $\gamma_{n}^{i} \in\left(\epsilon^{i}, \frac{2\left\|A^{i} x_{n}-T_{\mu}^{Q^{i}} A^{i} x_{n}\right\|^{2}}{\left\|\left(A^{i}\right)^{*} J_{E}\left(A^{i} x_{x_{n}}-T_{\mu}^{M^{i}} A^{i} x_{n}\right)\right\|^{2}}-\epsilon^{i}\right)$, if $T_{\mu}^{Q^{i}} A^{i} x_{n} \neq A^{i} x_{n}$; otherwise $\gamma_{n}^{i}=\gamma^{i}$ ( $\gamma^{i}$ being any nonnegative real number). Let $f_{n}: H \rightarrow H$ be a sequence of $\rho_{n}$-contractive mappings with $0<\underline{\rho} \leq \rho_{n} \leq \bar{\rho}<1$ such that $\left\{f_{n}(x)\right\}$ is uniformly convergent for any $x \in D$, where $D$ is any bounded subset of $H$. For arbitrary $x_{1} \in H$, define the iterative sequence $\left\{x_{n}\right\}$ by

$$
\left\{\begin{array}{l}
w_{n}^{i}=x_{n}-\gamma_{n}\left(A^{i}\right)^{*} J_{E}\left(A^{i} x_{n}-T_{\mu}^{Q^{i}} A^{i} x_{n}\right), \quad i=1,2, \ldots, I, \\
w_{n}=\eta_{n}^{1} x_{n}+\sum_{i=2}^{I} \eta_{n}^{i} \prod_{t=1}^{i-1}\left(1-\eta_{n}^{t}\right) w_{n}^{i-1}+\prod_{i=1}^{I}\left(1-\eta_{n}^{i}\right) w^{I}, \\
y_{n}=J_{\lambda_{n}}^{M}\left(I-\lambda_{n} N\right) w_{n}, \\
x_{n+1}=\beta_{n}^{1} f_{n}\left(x_{n}\right)+\left(1-\beta_{n}^{1}\right)\left[\beta_{n}^{2} x_{n}+\left(1-\beta_{n}^{2}\right)\left(v u_{n}+(1-v) y_{n}\right)\right],
\end{array}\right.
$$


where $u_{n} \in P_{S} x_{n}:=\left\{u_{n} \in S x_{n}:\left\|u_{n}-x_{n}\right\|=d\left(x_{n}, S x_{n}\right)\right\}, \mu>0, v \in(\theta, 1), 0<\lambda \leq \lambda_{n}<2 \alpha$, $\left\{\beta_{n}^{1}\right\}_{n=1}^{\infty},\left\{\beta_{n}^{2}\right\}_{n=1}^{\infty}$ and $\left\{\eta_{n}^{i}\right\}_{n=1}^{\infty}$ are real sequences in $(0,1)$ satisfying the following conditions:

(i) $\lim _{n \rightarrow \infty} \beta_{n}^{1}=0$ and $\sum_{n=1}^{\infty} \beta_{n}^{1}=\infty$;

(ii) $0<\epsilon_{1} \leq \beta_{n}^{2} \leq 1-\theta$;

(iii) $\liminf _{n \rightarrow \infty} \eta_{n}^{i} \prod_{t=1}^{i-1}\left(1-\eta_{n}^{t}\right)>0, i=2,3, \ldots,(I-1)$ and $\liminf _{n \rightarrow \infty} \prod_{t=1}^{I}\left(1-\eta_{n}^{t}\right)>0$.

Then the sequence $\left\{x_{n}\right\}$ converges strongly to an element of $\Upsilon$.

\section{Application to finite family split convex minimization problems}

Let $F^{j}: H \rightarrow \mathbb{R}$ be a finite family of convex and continuously differentiable functions, $G_{1}^{j}$ : $H \rightarrow(-\infty,+\infty]$ and $G_{2}^{i}: E \rightarrow(-\infty,+\infty]$ be two finite families of proper convex and lower semi-continuous functions. Then, for each $i=1,2, \ldots, I$ and $j=1,2, \ldots, J$, the gradient $\nabla F^{j}$ of $F^{j}$ is monotone and continuous, and the subdifferentials $\partial G_{1}^{j}: H \rightarrow 2^{H}$ of $G_{1}^{j}$ and $\partial G_{2}^{i}$ : $E \rightarrow 2^{E^{*}}$ of $G_{2}^{i}$ are maximal monotone (see [33]). Furthermore,

$$
F^{j}\left(x^{*}\right)+G_{1}^{j}\left(x^{*}\right)=\min _{x \in H}\left[F^{j}(x)+G_{1}^{j}(x)\right] \Leftrightarrow 0 \in \nabla F^{j}\left(x^{*}\right)+\partial G_{1}^{j}\left(x^{*}\right), \quad j=1,2, \ldots, J,
$$

and

$$
G_{2}^{i}\left(y^{*}\right)=\min _{y \in E} G_{2}^{i}(y) \quad \Leftrightarrow \quad 0 \in \partial G_{2}^{i}\left(y^{*}\right), \quad i=1,2, \ldots, I .
$$

Let us consider the following finite family of split convex minimization problem: Find

$$
x^{*} \in F\left(S^{k}\right) \text { such that } F^{j}\left(x^{*}\right)+G_{1}^{j}\left(x^{*}\right)=\min _{x \in H}\left[F^{j}(x)+G_{1}^{j}(x)\right]
$$

and

$$
y^{*}=A^{i} x^{*} \in E \text { such that } G_{2}^{i}\left(x^{*}\right)=\min _{y \in E} G_{2}^{i}(y),
$$

where $i=1,2, \ldots, I, j=1,2, \ldots, J, k=1,2, \ldots, K, A^{i}: H \rightarrow E$ is a bounded linear operator for each $i, F^{j}, G_{1}^{j}$ and $G_{2}^{i}$ are as defined above, $S^{k}: H \rightarrow P(H)$ is a finite family of multivalued type-one demicontractive mappings. Suppose the solution set of problem (4.1)-(4.1) is $\Omega$, then setting $M^{j}=\partial G_{1}^{j}, Q^{i}=\partial G_{2}^{i}$ and $N^{j}=\nabla F^{j}$ in Theorem 3.2, we obtain the following new result for approximating a common solution of finite family of split convex minimization problems and fixed point problem for a finite family of multivalued typeone demicontractive mappings.

Theorem 4.1 Let $H$ be a real Hilbert space and $E$ be a real uniformly convex and uniformly smooth Banach space. Let $j=1,2, \ldots, J, i=1,2, \ldots, I, k=1,2, \ldots, K$, for some $J, I, K \in \mathbb{N}$. For each $i, j, k$, let $A^{i}: H \rightarrow E$ be a bounded linear operator and $\left(A^{i}\right)^{*}: E^{*} \rightarrow H$ be the adjoint of $A^{i}$, let $G_{1}^{j}: H \rightarrow(-\infty,+\infty]$ and $G_{2}^{i}: E \rightarrow(-\infty,+\infty]$ be proper convex and lower semicontinuous functions, $F^{j}: H \rightarrow \mathbb{R}$ be a convex and continuously differentiable function such that $\nabla F^{j}$ is $\frac{1}{\alpha}$-Lipschitz continuous, and $S^{k}: H \rightarrow P(H), k=1,2, \ldots, K$ be a finite family of multivalued type-one demicontractive mappings with coefficient $\theta^{k} \in[0,1)$ such that $\left(I-S^{k}\right)$ is demiclosed at zero and $\Omega \neq \emptyset$. Let the step size $\gamma_{n}^{i}$ be chosen such that, for some $\epsilon^{i}>0, \gamma_{n}^{i} \in\left(\epsilon^{i}, \frac{2 \| A^{i} x_{n}-T_{\mu^{i}}^{\partial G_{2}^{i} A^{i} x_{n} \|}}{\left\|\left(A^{i}\right)^{*} J_{E}\left(A^{i} x_{n}-T_{\mu^{i}}{ }^{\partial} G_{2}^{i} x_{n}\right)\right\|}{ }^{2}-\epsilon\right)$, if $T_{\mu^{i}}^{\partial G^{i}} A^{i} x_{n} \neq A^{i} x_{n}$; otherwise $\gamma_{n}^{i}=\gamma^{i}\left(\gamma^{i}\right.$ being 
any nonnegative real number). Let $f_{n}: H \rightarrow H$ be a sequence of $\rho_{n}$-contractive mappings with $0<\underline{\rho} \leq \rho_{n} \leq \bar{\rho}<1$ such that $\left\{f_{n}(x)\right\}$ is uniformly convergent for any $x \in D$, where $D$ is any bounded subset of H. For arbitrary $x_{1} \in H$, define the iterative sequence $\left\{x_{n}\right\}$ by

$$
\left\{\begin{array}{l}
w_{n}^{i}=x_{n}-\gamma_{n}^{i}\left(A^{i}\right)^{*} J_{E}\left(A^{i} x_{n}-T_{\mu^{i}}^{\partial G_{2}^{i}} A^{i} x_{n}\right), \quad i=1,2, \ldots, I, \\
w_{n}=\eta_{n}^{1} x_{n}+\sum_{i=2}^{I} \eta_{n}^{i} \prod_{t=1}^{i-1}\left(1-\eta_{n}^{t}\right) w_{n}^{i-1}+\prod_{t=1}^{I}\left(1-\eta_{n}^{t}\right) w_{n}^{I}, \\
y_{n}^{j}=J_{\lambda_{n}^{j}}^{\partial G_{1}^{j}}\left(I-\lambda_{n}^{j} \nabla F^{j}\right) w_{n}, \quad j=1,2, \ldots, J, \\
y_{n}=\alpha_{n}^{1} w_{n}+\sum_{j=2}^{J} \alpha_{n}^{j} \prod_{t=1}^{j-1}\left(1-\alpha_{n}^{t}\right) y_{n}^{j-1}+\prod_{t=1}^{J}\left(1-\alpha_{n}^{t}\right) y_{n}^{J}, \\
u_{n}=\zeta_{n}^{1} x_{n}+\sum_{k=2}^{K} \zeta_{n}^{k} \prod_{t=1}^{k-1}\left(1-\zeta_{n}^{t}\right) u_{n}^{k-1}+\prod_{t=1}^{K}\left(1-\zeta_{n}^{t}\right) u_{n}^{K}, \\
x_{n+1}=\beta_{n}^{1} f_{n}\left(x_{n}\right)+\left(1-\beta_{n}^{1}\right)\left[\beta_{n}^{2} x_{n}+\left(1-\beta_{n}^{2}\right)\left(v u_{n}+(1-v) y_{n}\right)\right],
\end{array}\right.
$$

where $u_{n}^{k} \in P_{S^{k}} x_{n}:=\left\{u_{n}^{k} \in S^{k} x_{n}:\left\|u_{n}^{k}-x_{n}\right\|=d\left(x_{n}, S^{k} x_{n}\right)\right\}, k=1,2, \ldots, K, \mu>0, v \in(0,1)$, $0<\lambda^{j} \leq \lambda_{n}^{j}<2 \alpha^{j},\left\{\beta_{n}^{1}\right\}_{n=1}^{\infty},\left\{\beta_{n}^{2}\right\}_{n=1}^{\infty},\left\{\beta_{n}^{3}\right\}_{n=1}^{\infty},\left\{\alpha_{n}^{j}\right\}_{n=1}^{\infty},\left\{\eta_{n}^{i}\right\}_{n=1}^{\infty}$ and $\left\{\zeta_{n}^{k}\right\}_{n=1}^{\infty}$ are real sequences in $(0,1)$ satisfying the following conditions:

(i) $\lim _{n \rightarrow \infty} \beta_{n}^{1}=0$ and $\sum_{n=1}^{\infty} \beta_{n}^{1}=\infty$;

(ii) $0<\epsilon_{1} \leq \beta_{n}^{2}$;

(iii) $\zeta_{n}^{1} \geq \max \left\{\theta^{k}, k=1,2, \ldots, K\right\}, \liminf _{n \rightarrow \infty} \zeta_{n}^{k} \prod_{t=1}^{k-1}\left(1-\zeta_{n}^{t}\right)\left(\zeta_{n}^{1}-\theta^{k-1}\right)>0$,

$$
k=2,3, \ldots,(K-1) \text {; }
$$

(iv) $\liminf _{n \rightarrow \infty} \prod_{t=1}^{K}\left(1-\zeta_{n}^{t}\right)\left(\zeta_{n}^{1}-\theta^{K}\right)>0$;

(v) $\liminf _{n \rightarrow \infty} \eta_{n}^{i} \prod_{t=1}^{i-1}\left(1-\eta_{n}^{t}\right)>0, i=2,3, \ldots,(I-1)$ and $\liminf _{n \rightarrow \infty} \prod_{t=1}^{I}\left(1-\eta_{n}^{t}\right)>0$;

(vi) $\liminf _{n \rightarrow \infty} \alpha_{n}^{j} \prod_{t=1}^{j-1}\left(1-\alpha_{n}^{t}\right)>0, j=2,3, \ldots,(J-1)$ and $\liminf _{n \rightarrow \infty} \prod_{t=1}^{J}\left(1-\alpha_{n}^{t}\right)>0$.

Then the sequence $\left\{x_{n}\right\}$ converges strongly to $\Omega$.

\section{Numerical example}

We now give a numerical example of Algorithm (3.1) to show its performance. Let $H=\mathbb{R}^{2}$ and $E=\mathbb{R}^{4}$ (be endowed with the euclidean norm). Let $I=5, J=3$ and $K=4$. Then define $M^{j}: \mathbb{R}^{2} \rightarrow \mathbb{R}^{2}$ by

$$
M^{j}(x)=\left(j x_{1}-3 j x_{2}, 3 j x_{1}+j x_{2}\right), \quad j=1,2,3 .
$$

Clearly, $M^{j}$ is maximal monotone for each $j$. Thus, for each $x \in \mathbb{R}^{2}$, we compute the resolvent of $M^{j}$ as follows:

$$
\begin{aligned}
J_{\lambda_{n}^{j}}^{M^{j}}(x) & =\left(\left[\begin{array}{ll}
1 & 0 \\
0 & 1
\end{array}\right]+\left[\begin{array}{cc}
j \lambda_{n}^{j} & -3 j \lambda_{n}^{j} \\
3 j \lambda_{n}^{j} & j \lambda_{n}^{j}
\end{array}\right]\right)^{-1}\left[\begin{array}{l}
x_{1} \\
x_{2}
\end{array}\right] \\
& =\frac{1}{1+2 j \lambda_{n}^{j}+10 j^{2}\left(\lambda_{n}^{j}\right)^{2}}\left[\begin{array}{cc}
1+j \lambda_{n}^{j} & 3 j \lambda_{n}^{j} \\
-3 j \lambda_{n}^{j} & 1+j \lambda_{n}^{j}
\end{array}\right]\left[\begin{array}{l}
x_{1} \\
x_{2}
\end{array}\right],
\end{aligned}
$$

which implies that

$$
J_{\lambda_{n}^{j}}^{M^{j}}(x)=\left(\frac{\left(1+j \lambda_{n}^{j}\right) x_{1}+3 j \lambda_{n}^{j} x_{2}}{1+2 j \lambda_{n}^{j}+10 j^{2}\left(\lambda_{n}^{j}\right)^{2}}, \frac{\left(1+j \lambda_{n}^{j}\right) x_{2}-3 j \lambda_{n}^{j} x_{1}}{1+2 j \lambda_{n}^{j}+10 j^{2}\left(\lambda_{n}^{j}\right)^{2}}\right) .
$$


Also, we define $Q^{i}: \mathbb{R}^{4} \rightarrow \mathbb{R}^{4}$ by $Q^{i}(x)=\left(4 i x_{1}, 4 i x_{2}, 4 i x_{3}, 4 i x_{4}\right), i=1,2,3,4,5$. Then $Q^{i}$ is maximal monotone. In a similar way, we obtain the resolvent of $Q^{i}$ as

$$
T_{\mu^{i}}^{Q^{i}}(x)=\left(\frac{x_{1}}{4 i \mu^{i}+1}, \frac{x_{2}}{4 i \mu^{i}+1}, \frac{x_{3}}{4 i \mu^{i}+1}, \frac{x_{4}}{4 i \mu^{i}+1}\right)
$$

Let $A^{i}: \mathbb{R}^{2} \rightarrow \mathbb{R}^{4}$ be defined by $A^{i}(x)=\left(-3 i x_{1}+4 i x_{2}, i x_{1}+5 i x_{2}, 3 i x_{1}-2 i x_{2}, 2 i x_{1}-3 i x_{2}\right)$, $f_{n}: \mathbb{R}^{2} \rightarrow \mathbb{R}^{2}$ be defined by $f_{n}(x)=\frac{4}{5} x \forall n \geq 1$, and $S^{k}: \mathbb{R}^{2} \rightarrow \mathbb{R}^{2}$ be defined by $S^{k}(x)=$ $-\left(\frac{2 k+1}{2}\right)\left(x_{1}, x_{2}\right), k=1,2,3,4$, then $S^{k}$ is a $\theta^{k}$-demicontractive mapping with $\theta^{k}=\frac{4 k^{2}+4 k-3}{(2 k+3)^{2}}$ for each $k=1,2,3,4$. Thus, $\theta=\frac{77}{121}$. Now, define $N^{j}: \mathbb{R}^{2} \rightarrow \mathbb{R}^{2}$ by $N(x)=\left(4 j x_{1}, 4 j x_{2}\right), j=1,2,3$. Then $N$ is an $\alpha^{j}$-inverse strongly monotone mapping with $\alpha^{j}=\frac{1}{4 j}$. Take $v=\frac{1}{2}, \beta_{n}^{1}=\frac{1}{n+1}$, $\beta_{n}^{2}=\frac{n+1}{5 n}, \lambda_{n}^{j}=\frac{n}{4 j n+1}, \eta_{n}^{i}=\frac{2 i n+3}{4+6 i n}, \alpha_{n}^{j}=\frac{n+1}{4 j n+2}, \zeta_{n}^{k}=\frac{77 n+2}{121 k n+1}$ and $\gamma_{n}^{i} \in\left(\epsilon^{i}, \frac{2\left\|A^{i} x_{n}-T_{\mu^{i}}^{Q^{i}} A^{i} x_{n}\right\|^{2}}{\left\|\left(A^{i}\right)^{T}\left(A^{i} x_{n}-T_{\mu^{i}}^{M^{i}} A^{i} x_{n}\right)\right\|^{2}}-\right.$ $\epsilon^{i}$ ), if $T_{\mu^{i}}^{Q^{i}} A^{i} x_{n} \neq A^{i} x_{n}$; otherwise $\gamma_{n}^{i}=\gamma^{i}$ ( $\gamma^{i}$ being any nonnegative real number). Then conditions (i)-(vi) of Theorem 3.2 are satisfied. Hence, we consider the following cases for $\mu^{i}$ and our initial points $x_{1}$ (see Fig. 1).
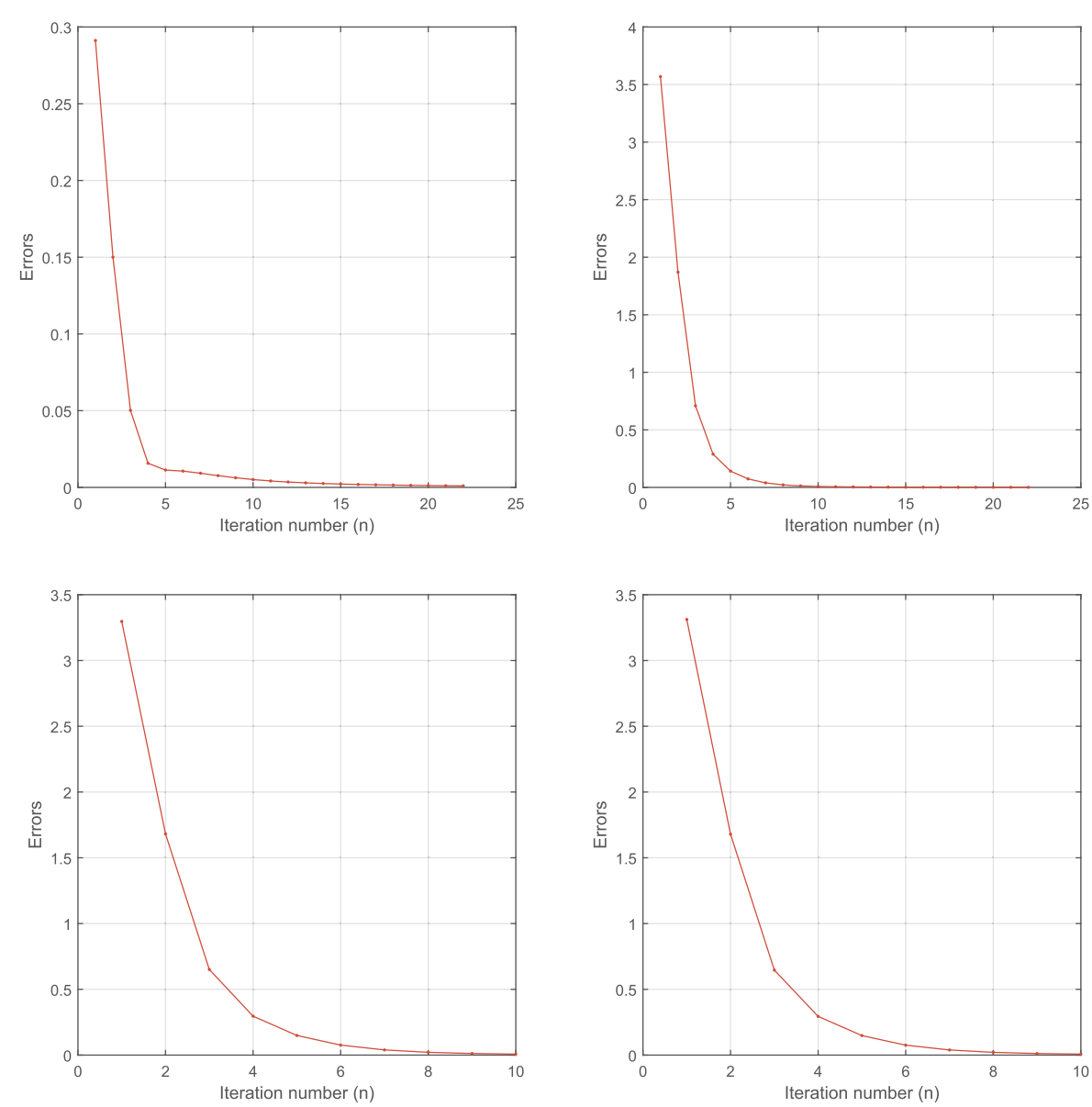

Figure 1 Errors vs. iteration numbers (n): Case I(a) (top left); Case I(b) (top right); Case II(a) (bottom left); Case II(b) (bottom right) 
Case I:

(a) Take $x_{1}=(0.5,-0.25)^{T}$ and $\mu^{i}=\mu=\frac{8}{10}$.

(b) Take $x_{1}=(-5,4)^{T}$ and $\mu^{i}=\mu=\frac{8}{10}$.

Case II:

(a) Take $x_{1}=(-5,3)^{T}$ and $\mu^{i}=\mu=10$.

(b) Take $x_{1}=(-5,3)^{T}$ and $\mu^{i}=\mu=1000$.

\section{Conclusion}

A new viscosity-type iteration process that does not involve the construction of either $C_{n}$ or $Q_{n}$ is introduced for approximating a common solution of a finite family of split variational inclusion problem and fixed point problem for a finite family of type-one demicontractive mappings between a Hilbert space and a Banach space. Furthermore, our choice of stepsize in Algorithm (3.1) does not depend on the norm of the bounded linear operator $A$ which is very difficult to compute in general as illustrated in Theorem 1.6. Moreover, we established a strong convergence theorem for approximating a solution of the aforementioned problem and our control sequences do not require the imposition of the condition that the sum $=1$. Some applications and numerical experiments of the established results are given to further illustrate the applicability of our results.

\section{Acknowledgements}

The second author acknowledges with thanks the bursary and financial support from Department of Science and Technology and National Research Foundation, Republic of South Africa Center of Excellence in Mathematical and Statistical Sciences (DST-NRF COE-MaSS) Post Doctoral Bursary (2018). Opinions expressed and conclusions arrived are those of the authors and are not necessarily to be attributed to the CoE-MaSS.

\section{Funding}

This research was supported by CoE-MaSS BA2018-012.

Availability of data and materials

All data generated or analysed during this study are included in this published article.

\section{Competing interests}

The authors declare that they have no competing interests.

Authors' contributions

The main idea of this paper was proposed by FOI, Cl prepared the manuscript initially and performed all the steps of the proofs in this research. CCO assisted in proofreading. All authors read and approved the final manuscript.

\section{Author details}

${ }^{1}$ School of Mathematics, Statistics and Computer Science, University of Kwazulu-Natal, Durban, South Africa. ${ }^{2}$ DST-NRF Center of Excellence in Mathematical and Statistical Sciences (CoE-Mass), Johannesburg, South Africa. ${ }^{3}$ Department of Mathematics, University of Nigeria, Nsukka, Nigeria.

\section{Publisher's Note}

Springer Nature remains neutral with regard to jurisdictional claims in published maps and institutional affiliations.

Received: 1 April 2019 Accepted: 6 September 2019 Published online: 23 September 2019

\section{References}

1. Ahmad, R., Ansari, Q.H.: An iterative algorithm for generalized nonlinear variational inclusions. Appl. Math. Lett. 13, $23-26(2000)$

2. Ahmad, R., Ansari, Q.H., Irfan, S.S.: Generalized variational inclusions and generalized resolvent equations in Banach spaces. Comput. Math. Appl. 29, 1825-1835 (2005)

3. Aoyama, K., Kohsaka, F.: Existence of fixed points of firmly nonexpansive-like mappings in Banach spaces. Fixed Point Theory Appl. 2010, Article ID 512751 (2010). https://doi.org/10.1155/2010/512751

4. Brouwer, L.E.J.: Uber Abbildung von Mannigfaltigkeiten. Math. Ann. 71(4), 598 (1912)

5. Browder, F.E.: Nonlinear maximal monotone operators in Banach spaces. Math. Ann. 175, 89-113 (1968) 
6. Byrne, C.: Iterative oblique projection onto convex sets and the split feasibility problem. Inverse Probl. 18, 441-453 (2002)

7. Byrne, C.: A unified treatment for some iterative algorithms in signal processing and image reconstruction. Inverse Probl. 20, 103-120 (2004)

8. Byrne, C., Censor, Y., Gibali, A., Reich, S.: Weak and strong convergence of algorithms for the split common null point problem. J. Nonlinear Convex Anal. 13, 759-775 (2012)

9. Censor, Y., Elfving, T.: A multiprojection algorithm using Bregman projections in product space. Numer. Algorithms 8 , 221-239 (1994)

10. Chang, S.S.: Existence and approximation of solutions for set-valued variational inclusion in Banach spaces. Nonlinear Anal. 47, 583-594 (2001)

11. Chang, S.S., Wen, C.F., Yao, J.C.: Common zero point for a finite family of inclusion problems of accretive mappings in Banach spaces. Optimization 67, 1183-1196 (2018)

12. Chidume, C.E.: Geometric Properties of Banach Spaces and Nonlinear Iterations. Springer Verlag Series, Lecture Notes in Mathematics (2009). ISBN:978-1-84882-189-7

13. Chidume, C.E., Ezeora, J.: Krasnoselskii-type algorithm for family of multi-valued strictly pseudocontractive mappings. Fixed Point Theory Appl. 2014, 111 (2014)

14. Eslamian, M.: Hybrid method for equilibrium problems and fixed point problems of finite families of nonexpansive semigroups. Rev. R. Acad. Cienc. Exactas Fís. Nat., Ser. A Mat. 107(2), 299-307 (2003)

15. Ezeora, J.N., Izuchukwu, C.: Iterative approximation of solution of split variational inclusion problems. Filomat 32, 2921-2932 (2018)

16. Hendrickx, J.M., Olshevsky, A.: Matrix $P$-norms are NP-hard to approximate if $P \neq 1,2, \infty$. SIAM J. Matrix Anal. Appl. 31, 2802-2812 (2010)

17. Isiogugu, F.O.: Demiclosedness principle and approximation theorems for certain classes of multivalued mappings in Hilbert spaces. Fixed Point Theory Appl. 2013, 61 (2013)

18. Isiogugu, F.O., Izuchukwu, C., Okeke, C.C.: New iteration scheme for approximating a common fixed point of finite family of mappings. J. Math. (under review)

19. Isiogugu, F.O., Osilike, M.O.: Convergence theorems for new classes of multivalued hemicontractive-type mappings. Fixed Point Theory Appl. 2014, 93 (2014)

20. Isiogugu, F.O., Pillay, P., Baboolal, D.: Approximation of a common element of the set of fixed points of multi-valued type-one demicontractive-type mappings and the set of solutions of an equilibrium problem in Hilbert spaces. J. Nonlinear Convex Anal. 17(6), 1181-1197 (2016)

21. Isiogugu, F.O., Pillay, P., Osilike, M.O.: On approximation of fixed points of multi-valued quasi-nonexpansive mappings in Hilbert spaces. J. Nonlinear Convex Anal. 17(7), 1303-1310 (2016)

22. Izuchukwu, C.: A common solution of finite family of a new class of split monotone variational inclusion problems. Adv. Nonlinear Var. Inequal. 21(1), 83-104 (2018)

23. Izuchukwu, C., Isiogugu, F.O., Okeke, C.C.: A viscosity iterative technique for split variational inclusion and fixed point problems between a Hilbert space and a Banach space. J. Fixed Point Theory Appl. (2018). https://doi.org/10.1007/s11784-018-0632-4

24. Kazmi, K.R., Ali, R., Furkan, M.: Hybrid iterative method for split monotone variational inclusion problem and hierarchical fixed point problem for a finite family of nonexpansive mappings. Numer. Algorithms (2017). https://doi.org/10.1007/s11075-017-0448-0

25. Kazmi, K.R., Rizvi, S.H.: An iterative method for split variational inclusion problem and fixed point problem for a nonexpansive mapping. Optim. Lett. 8(3), 1113-1124 (2014). https://doi.org/10.1007/s11590-013-0629-2

26. Kimura, Y., Nakajo, K.: Strong convergence for a modified forward-backward splitting method in Banach spaces. J. Nonlinear Var. Anal. 3, 5-18 (2019)

27. Maingé, P.E.: Strong convergence of projected subgradient methods for nonsmooth and nonstrictly convex minimization. Set-Valued Anal. 16, 899-912 (2008)

28. Matsushita, S.-Y., Takahashi, W.: On the existence of zeroes of monotone operators in reflexive Banach spaces. J. Math. Anal. Appl. 323, 1354-1364 (2006)

29. Moudafi, A.: Split monotone variational inclusions. J. Optim. Theory Appl. 150, 275-283 (2011)

30. Nadler, S.B.: Multivalued contraction mappings. Pac. J. Math. 30, 475-488 (1969)

31. Promluang, K., Kuman, P. Viscosity approximation method for split common null point problems between Banach spaces and Hilbert spaces. J. Inform. Math. Sci. 9(1), 27-44 (2017)

32. Qin, X., Yao, J.C.: Projection splitting algorithms for nonself operators. J. Nonlinear Convex Anal. 18, 925-935 (2017)

33. Rockafellar, R.T:: On the maximality of sums of nonlinear monotone operators. Trans. Am. Math. Soc. 149, 75-288 (1970)

34. Shang, M.: A descent-like method for fixed points and split conclusion problems. J. Appl. Numer. Optim. 1, 91-101 (2019)

35. Shehu, Y., Ogbuisi, F.U.: An iterative method for solving split monotone variational inclusion and fixed point problems Rev. R. Acad. Cienc. Exactas Fís. Nat., Ser. A Mat. 110(2), 503-518 (2016). https://doi.org/10.1007/s13398-015-0245-3

36. Takahashi, W. Strong convergence theorem for a finite family of demimetric mappings with variational inequality problems in a Hilbert space. Jpn. J. Ind. Appl. Math. 34, 41-57 (2017)

37. Takahashi, W., Wen, C.F., Yao, J.C.: The shrinking projection method for a finite family of demimetric mappings with variational inequality problems in a Hilbert space. Fixed Point Theory 19, 407-419 (2018)

38. Takahashi, W., Yao, J.C.: Strong convergence theorems by hybrid methods for the split common null point problem in Banach spaces. Fixed Point Theory Appl. 2015, 87 (2015)

39. Xu, H.K.: Iterative algorithm for nonlinear operators. J. Lond. Math. Soc. 66(2), 240-256 (2002)

40. Yao, Y., Qin, X., Yao, J.C.: Convergence analysis of an inertial iterate for the proximal split feasibility problem. J. Nonlinear Convex Anal. 20, 489-498 (2019)

41. Yao, Y.-H., Liou, Y.-C., Yao, J.-C.: Iterative algorithms for the split variational inequality and fixed point problems under nonlinear transformations. J. Nonlinear Sci. Appl. 10, 843-854 (2017)

42. Yao, Y.H., Agarwal, R.P., Postolache, M., Liou, Y.C.: Algorithms with strong convergence for the split common solution of the feasibility problem and fixed point problem. Fixed Point Theory Appl. 2014, 183 (2014) 
43. Zegeye, H., Shahzad, N., Yao, Y.-H.: Minimum-norm solution of variational inequality and fixed point problem in Banach spaces. Optimization 64, 453-471 (2015)

44. Zhou, H.Y.: Convergence theorems of fixed points for strict pseudo-contractions in Hilbert spaces. Nonlinear Anal. 69(2), 456-462 (2008)

Submit your manuscript to a SpringerOpen ${ }^{\circ}$ journal and benefit from:

- Convenient online submission

$\checkmark$ Rigorous peer review

Open access: articles freely available online

- High visibility within the field

- Retaining the copyright to your article

Submit your next manuscript at $\gg$ springeropen.com 